\title{
Phylogenetic Analyses of Basal Angiosperms Based on Nine Plastid, Mitochondrial, and Nuclear Genes
}

\section{Citation}

Qiu, Yin-Long, Olena Dombrovska, Jungho Lee, Libo Li, Barbara A. Whitlock, Fabiana Bernasconi-Quadroni, Joshua S. Rest, et al. 2005. Phylogenetic analyses of basal angiosperms based on nine plastid, mitochondrial, and nuclear genes. International Journal of Plant Sciences 166(5): 815-842.

\section{Published Version}

http://dx.doi.org/10.1086/431800

\section{Permanent link}

http://nrs.harvard.edu/urn-3:HUL.InstRepos:2710479

\section{Terms of Use}

This article was downloaded from Harvard University's DASH repository, and is made available under the terms and conditions applicable to Other Posted Material, as set forth at http:// nrs.harvard.edu/urn-3:HUL.InstRepos:dash.current.terms-of-use\#LAA

\section{Share Your Story}

The Harvard community has made this article openly available.

Please share how this access benefits you. Submit a story.

Accessibility 
Int. J. Plant Sci. 166(5):815-842. 2005.

(c) 2005 by The University of Chicago. All rights reserved.

$1058-5893 / 2005 / 16605-0012 \$ 15.00$

\title{
PHYLOGENETIC ANALYSES OF BASAL ANGIOSPERMS BASED ON NINE PLASTID, MITOCHONDRIAL, AND NUCLEAR GENES
}

\author{
Yin-Long Qiu, ${ }^{1, *}+\neq \neq$ Olena Dombrovska, ${ }^{*} \uparrow \neq \neq$ Jungho Lee, ${ }^{2, \dagger} \neq$ Libo Li, ${ }^{*} \dagger$ Barbara A. Whitlock, ${ }^{3, \dagger}$ Fabiana Bernasconi-Quadroni, $\neq$ \\ Joshua S. Rest, ${ }^{4, *}$ Charles C. Davis, ${ }^{*}$ Thomas Borsch, $\S$ Khidir W. Hilu, I Susanne S. Renner, ${ }^{5}$ \# Douglas E. Soltis, ${ }^{* *}$ \\ Pamela S. Soltis, †† Michael J. Zanis, ${ }^{6} \neq \ddagger$ Jamie J. Cannone, §§ Robin R. Gutell,§§ Martyn Powell,\|l\| \\ Vincent Savolainen, \|\| $\mid$ Lars W. Chatrou,\#\# and Mark W. Chase \|\|

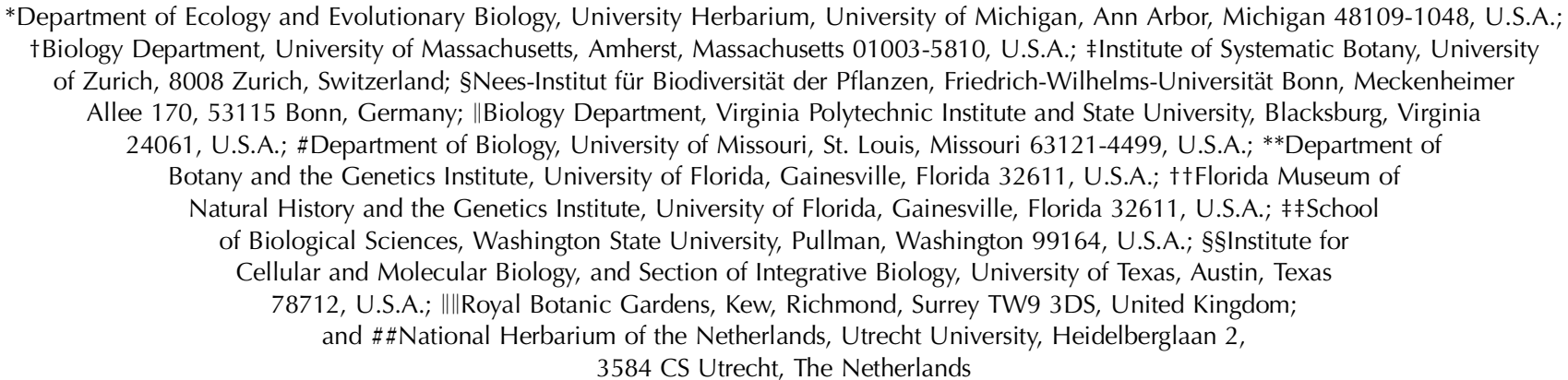

DNA sequences of nine genes (plastid: atpB, matK, and $r b c L$; mitochondrial: atp1, matR, mtSSU, and mtLSU; nuclear: $18 \mathrm{~S}$ and 26S rDNAs) from 100 species of basal angiosperms and gymnosperms were analyzed using parsimony, Bayesian, and maximum likelihood methods. All of these analyses support the following consensus of relationships among basal angiosperms. First, Amborella, Nymphaeaceae, and Austrobaileyales are strongly supported as a basal grade in the angiosperm phylogeny, with either Amborella or Amborella and Nymphaeales as sister to all other angiosperms. An examination of nucleotide substitution patterns of all nine genes ruled out any possibility of analytical artifacts because of RNA editing and GC-content bias in placing these taxa at the base of the angiosperm phylogeny. Second, Magnoliales are sister to Laurales and Piperales are sister to Canellales. These four orders together constitute the magnoliid clade. Finally, the relationships among Ceratophyllum, Chloranthaceae, monocots, magnoliids, and eudicots are resolved in different ways in various analyses, mostly with low support. Our study indicates caution in total evidence approaches in that some of the genes employed (e.g., mtSSU, mtLSU, and nuclear 26S rDNA) added signal that conflicted with the other genes in resolving certain parts of the phylogenetic tree.

Keywords: basal angiosperms, Amborella, magnoliids, multigene analysis, synapomorphic substitutions, phylogeny.

\section{Introduction}

The past 20 years have witnessed significant progress in our understanding of the phylogeny of basal angiosperms from analyses of molecular and nonmolecular data (Dahlgren and Bremer 1985; Donoghue and Doyle 1989; Loconte

${ }^{1}$ Author for correspondence; e-mail ylqiu@umich.edu.

2 Current address: School of Biological Sciences, Seoul National University, Shillim, Kwanak, Seoul, South Korea 151-747.

${ }^{3}$ Current address: Department of Biology, University of Miami, Miami, Florida 33124-0421, U.S.A.

${ }^{4}$ Current address: Department of Ecology and Evolution, University of Chicago, Chicago, Illinois 60637, U.S.A.

${ }^{5}$ Current address: Department of Biology, Ludwig Maximilians University Munich, Munich, Germany.

${ }^{6}$ Current address: Division of Biological Sciences, University of California, San Diego, La Jolla, California 92093, U.S.A.

Manuscript received November 2004; revised manuscript received May 2005. and Stevenson 1991; Martin and Dowd 1991; Hamby and Zimmer 1992; Taylor and Hickey 1992; Chase et al. 1993; Qiu et al. 1993, 1999, 2000, 2001; Soltis et al. 1997, 2000; Nandi et al. 1998; Hoot et al. 1999; Mathews and Donoghue 1999, 2000; Parkinson et al. 1999; Renner 1999; Soltis et al. 1999a; Barkman et al. 2000; Doyle and Endress 2000; Graham and Olmstead 2000b; Savolainen et al. 2000; Nickrent et al. 2002; Zanis et al. 2002, 2003; Borsch et al. 2003; Hilu et al. 2003; Löhne and Borsch 2005). Specifically, it has become increasingly clear that Amborella, Nymphaeaceae, and Austrobaileyales (sensu APG II 2003) represent the earliestdiverging lineages of extant angiosperms. Furthermore, the magnoliids (sensu APG II 2003; see Qiu et al. 1993 for a review of the history of this term) have been identified as a monophyletic group in some analyses (Qiu et al. 1999, 2000; Zanis et al. 2002, 2003; Hilu et al. 2003), but their monophyly (Savolainen et al. 2000; Soltis et al. 2000) and especially relationships among their member orders (Magnoliales, Laurales, Piperales, and Canellales) need further evaluation 
and resolution. Finally, all angiosperms excluding Amborella, Nymphaeaceae, and Austrobaileyales can be divided into five clades: Ceratophyllum, Chloranthaceae, magnoliids, monocots, and eudicots (tricolpates sensu Judd and Olmstead 2004; see also Walker and Doyle 1975; Crane 1989; Donoghue and Doyle 1989; Doyle and Hotton 1991; Chase et al. 1993). Relationships among these five lineages, however, are best interpreted as unresolved at present because analyses with different taxon and character-sampling schemes and phylogenetic methods have produced conflicting topologies that are generally only weakly supported (Barkman et al. 2000; Soltis et al. 2000; Zanis et al. 2002, 2003; Hilu et al. 2003).

Despite progress, more work is needed to further clarify relationships among basal angiosperms. In this study, we add sequence data of four new genes to a five-gene matrix assembled earlier (Qiu et al. 1999, 2000) and conduct parsimony, Bayesian, and maximum likelihood (ML) analyses to address several issues. First, we attempt to show that placement of Amborella, Nymphaeaceae, and Austrobaileyales at the base of angiosperm phylogeny is free of any analytical artifact. This is especially important in light of recent analyses of the entire plastid genome sequences of Amborella and Nymphaea that do not support them as basalmost angiosperms (Goremykin et al. 2003a, 2003b, 2004; but see Soltis and Soltis 2004; Soltis et al. 2004; Stefanovic et al. 2004). Second, we aim to evaluate the monophyly of magnoliids and to resolve the relationships among their members: Magnoliales, Laurales, Piperales, Canellales. Finally, we wish to resolve relationships among Ceratophyllum, Chloranthaceae, magnoliids, monocots, and eudicots.

\section{Material and Methods}

We included 100 terminals from 98 genera, representing all major lineages of gymnosperms and basal angiosperms. Acorus and Ceratophyllum were the only two genera for which two species each were sampled. Only two families of basal angiosperms were not included, Gomortegaceae (Renner 1999) and Hydnoraceae (Nickrent et al. 2002), because of many missing data entries. Most of the terminals consist of sequences derived from a single species (and frequently the same DNA sample) and occasionally from different species of the same genus (tables 1,2). Eight gymnosperms covering all four extant lineages were used as outgroups.

The four new genes added in this study are: plastid matK (a group II intron-encoded maturase), mitochondrial SSU (small subunit) and LSU (large subunit) rDNAs, and nuclear $26 \mathrm{~S}$ rDNA. With the five genes from our earlier analyses (mitochondrial atp1 and matR, plastid $a t p B$ and $r b c L$, and nuclear $18 \mathrm{~S}$ rDNA), the total of nine genes used in this study represents a sampling of a large number of characters from each of the three plant genomes. Furthermore, these genes encompass diverse functions, including energy metabolism, carbohydrate synthesis, RNA processing, and protein synthesis.

DNA extraction and sequencing methods follow Qiu et al. (2000). All primer sequences used for amplifying and sequencing the genes are available from the corresponding author on request. All sequences of mtLSU were newly generated in this study, whereas approximately half of the se- quences were generated by us for mtSSU, mat $K$, and nuclear $26 \mathrm{~S}$ rDNA. For the five genes used in Qiu et al. (1999), several new sequences were produced to fill the missing entries in that matrix. The orthologous atp1 was used to replace the copy we obtained earlier from Amborella (Qiu et al. 1999, 2000), which has been shown to be a xenolog horizontally transferred from an asterid (Barkman et al. 2000; Bergthorsson et al. 2003). For all nine genes we have taken sequences from GenBank when appropriate. Detailed source information for all sequences and correction to errors in table A1 of Qiu et al. (2000) are provided in tables 1 and 2. Of all taxa and all genes, only four taxa have missing data in one or two genes: Metasequoia ( $\mathrm{mtSSU}$ and matR), Hortonia (matR), and Dioscorea and Myristica (nu26S) (tables 1,2). Eight of the nine genes (all except mtSSU) were aligned using Clustal $\mathrm{X}$ (Thompson et al. 1997). Because of extraordinary length variation in several regions of $\mathrm{mtSSU}$, this gene was manually aligned with the alignment editor AE2 (developed by T. Macke; Larsen et al. 1993). Although these regions typically had minimal sequence identity that could not be aligned based on sequence alone, they usually had similar structural elements that facilitated the alignment of these sequences. In addition, all of the computer-generated alignments were manually adjusted with the MacClade 4.05 (Maddison and Maddison 2002) alignment editor. All of the aligned positions were used in the phylogenetic analyses. We also eliminated the positions in regions with significant length variations in the four rDNAs from the phylogenetic analyses of the ninegene matrix. These latter analyses yielded results not substantially different from those presented here (data not shown).

Three series of analyses were performed to address various issues. First, two separate matrices were assembled to reconstruct the overall phylogeny of basal angiosperms, one consisting of all nine genes and the other of five protein-coding genes. The decision to make a separate matrix using the five protein-coding genes was based on the following considerations: (1) all positions within the protein genes should evolve more independently than those of rDNAs, many of which evolve in a coupled fashion due to base pairing in stem regions in these genes (Soltis and Soltis 1998; Soltis et al. 1999b; O. Dombrovska and Y.-L. Qiu, unpublished data); (2) the protein-coding genes generally show fewer problems of paralogy and xenology compared to nuclear $18 \mathrm{~S}$ and $26 \mathrm{~S}$ rDNAs, for which nonorthologous copies were occasionally encountered; and (3) the protein-coding genes are free of alignment uncertainties compared to two mitochondrial rDNAs, which exhibit extraordinary length variations caused by insertions and deletions in a few regions. The parsimony, Bayesian, and maximum likelihood (ML) analyses were implemented separately on both matrices. To evaluate the informativeness of the two nuclear rDNAs further, the fiveprotein-gene matrix was combined with $18 \mathrm{~S}$ and $26 \mathrm{~S}$ rDNAs sequentially to form two more matrices. Only parsimony bootstrap analyses were conducted on these two matrices.

Second, three separate genome-specific matrices were constructed to address whether placement of Amborella, Nymphaeaceae, and Austrobaileyales as sisters to all other extant angiosperms is supported by data from the plastid, mitochondrial, and nuclear genomes separately. This type of analysis has only been conducted occasionally (Mathews and 
Donoghue 1999, 2000; Graham and Olmstead 2000b; Savolainen et al. 2000; Zanis et al. 2002). A robust understanding of organismal phylogeny should be based on evidence from each of the three plant genomes (Qiu and Palmer 1999) except in cases of hybridization and horizontal gene transfer. Only parsimony bootstrap analyses were conducted on these data sets.

Third, we investigated the types of substitutions that provided phylogenetic signal for identifying Amborella, Nymphaeaceae, and Austrobaileyales as the earliest-diverging lineages of extant angiosperms. For an issue as critical as the rooting of angiosperm phylogeny, merely having high bootstrap numbers from an analysis is not enough to gain confidence in the result (Soltis et al. 2004). Some poorly understood molecular evolutionary phenomena, such as RNA editing (Steinhauser et al. 1999; Kugita et al. 2003; Dombrovska and Qiu 2004) and GC-content bias (Steel et al. 1993), both of which can occur in a genome-wide, lineagespecific fashion, can generate substitutions that lead to spurious groupings in phylogenetic analyses. Hence, it is important that we understand the types of substitutions that are behind those high bootstrap percentages. We examined the nine-gene matrix visually and identified the sites that contain apparently synapomorphic changes that separate gymnosperms-AmborellaNymphaeaceae-Austrobaileyales from all other angiosperms. Sites were classified as apparently synapomorphic if they contained the same nucleotide in at least two of the four gymnosperm lineages (cycads, Ginkgo, conifer II [non-Pinaceae conifers], and Gnetales + Pinaceae; Bowe et al. 2000; Chaw et al. 2000) and at least two of the three basal angiosperm lineages (Amborella, Nymphaeaceae, and Austrobaileyales) but had a different and generally invariable nucleotide in all other angiosperms (hence a synapomorphy for euangiosperms, sensu Qiu et al. 1999). We then performed both a most parsimonious tree search and a parsimony bootstrap analysis with these sites removed to verify our identification. These synapomorphic substitutions were finally checked to determine if they could have been generated by RNA editing or GC-content bias. In addition, codon position and type of change (transition vs. transversion) were noted for these substitutions.

These last two series of analyses were designed to complement the analyses we performed earlier (Qiu et al. 1999, 2000, 2001), to ensure that the placement of Amborella, Nymphaeaceae, and Austrobaileyales as basal lineages is indeed based on historical signal recorded in the multiple genes from all three plant genomes rather than the result of yet poorly understood analytical artifacts. These analyses are particularly relevant in the ongoing debate over whether Amborella and Nymphaea are basal angiosperms (Goremykin et al. 2003a, 2003b, 2004; Soltis et al. 2004; Soltis and Soltis 2004; Stefanovic et al. 2004).

In parsimony searches we used equal weighting for all positions and character-state changes using PAUP* 4.0b10 (Swofford 1998). When searching for the shortest trees, a heuristic search was conducted using 1000 random taxon-addition replicates, one tree held at each step during stepwise addition, TBR branch swapping, steepest descent option off, MulTrees option on, and no upper limit of MaxTrees. For bootstrap analyses, 1000 resampling replicates were performed (except for the matrix of five protein genes plus two nuclear rDNAs where 5000 resampling replicates were used) with the same tree search procedure as described above except with simple taxon addition and the steepest descent option on.

For Bayesian and ML analyses, the optimal models of sequence evolution for the nine-gene and five-protein-gene data sets were estimated using ModelTest 3.6 (Posada and Crandall 1998) and DT-ModSel (Minin et al. 2003). The general time-reversible model (Rodríguez et al. 1990) including parameters for invariant sites and rate variation $(G T R+I+\Gamma)$ best fits both data sets and was used to conduct the analyses.

Bayesian analyses were performed using MrBayes version 3.0b4 (Huelsenbeck and Ronquist 2001). For the nine-gene matrix, the data were partitioned according to codon positions (first, second, and third, for protein genes only), genomes (plastid, mitochondrial, and nuclear), and gene types within a genome (rRNA vs. protein genes). For the five-protein-gene matrix, the data were partitioned according to codon positions and genomes. Calculations of likelihood for searches of both matrices were implemented under the GTR $+\mathrm{I}+\Gamma$ model of sequence evolution, assuming different stationary nucleotide frequencies. The posterior probability (PP) was estimated by sampling trees from the PP distribution using Metropolis coupled Markov Chain Monte Carlo methods. Two and four chains of 5,000,000 generations were run for the nine-gene matrix and five-protein-gene matrix, respectively. Chains were sampled every 100 generations. Likelihood scores converged on a stable value after 500,000 generations (the burn-in of the chain), and calculations of PP were based on the trees sampled after this generation.

Maximum likelihood analyses were performed separately on the nine-gene and five-protein-gene data sets using PHYML version 2.4.4 (Guindon and Gascuel 2003) under the optimal model of sequence evolution. For both data sets, the $\mathrm{GTR}+\mathrm{I}+\Gamma$ model was implemented with parameter values for the proportion of invariant sites (nine-gene $=0.19$, five-gene-protein $=0.21$ ) and the gamma distribution (ninegene $=0.43$, five-gene-protein $=0.68$ ) as estimated by ModelTest 3.6 and DT-ModSel. The optimal rate of nucleotide substitution and transition/transversion ratios was estimated from the data during ML searches. Maximum likelihood support values were similarly estimated from 100 bootstrap replicates in PHYML.

\section{Results}

For the nine-gene data set, which contained 26,990 aligned nucleotides, two islands with two and four shortest trees (length $=51,834$ steps; consistency index $[\mathrm{CI}]=0.47$; retention index $[\mathrm{RI}]=0.57)$ were found 259 and 315 times, respectively, out of 1000 random taxon-addition replicates in the parsimony search. One of the six trees is shown (fig. 1), with the nodes that are not present in the strict consensus of all six trees indicated by asterisks.

For the five-protein-gene data set, which contained 9351 aligned nucleotides, a single island of two shortest trees (length $=18,839$ steps; $\mathrm{CI}=0.42 ; \mathrm{RI}=0.59$ ) was found in all 1000 random taxon-addition replicates in the parsimony search. One of the two trees is shown (fig. 2), with the nodes 
Table 1

Vouchers, Contributors, GenBank Accession Numbers, and References for the Sequences Used in This Study

Family and species

Acoraceae:

Acorus calamus L.

Acorus gramineus Soland.

Alismataceae:

Alisma plantago-aquatica $\mathrm{L}$.

Alisma canaliculatum A. Br. \& Bouché

Amborellaceae:

Amborella trichopoda Baill.

Annonaceae:

Annona muricata L.

Cananga odorata (Lam.) Hook. f. \& Thomson

Araceae:

Orontium aquaticum L.

Spathiphyllum $\times$ 'Clevelandii'

Spathiphyllum floribundum (Lind.\& Andre) N.E.Br.

Spathiphyllum wallisii Hort.

Aristolochiaceae:

Aristolochia macrophylla Lam.

Aristolochia Pistolochia L.

Asarum canadense L.

Asarum yakusimense Masam.

Saruma henryi Oliv.

Thottea tomentosa Ding Hou

\section{Asparagaceae:}

Asparagus officinalis $\mathrm{L}$.

Asparagus filicinus

mt-SSU rDNA

mt-LSU rDNA

cp-matK
Parkinson et al. 1999; AF193976

Qiu 97131; JL/LL/YQ; DQ008668

Qiu 96177; JL/LL/YQ; DQ008669

Parkinson et al. 1999; AF193987

Qiu 90031; JL/LL/YQ; DQ008670

Chase 219, NCU; JL/LL/YQ; DQ008671

Qiu 97112; JL/LL/YQ DQ008672

Qiu 94140; JL/LL/YQ; DQ008673

Qiu 91019; JL/LL/YQ; DQ008674

Kuhlman sn, IND, Qiu 96018*; JL/LL/YQ;

DQ008676

Qiu 91018; JL/LL/YQ; DQ008677

Chase 1211, K; JL/LL/YQ;

DQ008675

Qiu 94063; JL/LL/YQ; DQ008678
Qiu 94052; OD/FBQ/YQ DQ008817

Qiu 97131; OD/FBQ/YQ; DQ008818

Qiu 96177; OD/FBQ/YQ; DQ008812

B. Hall sn, IND, Qiu 97123*; OD/FBQ/YQ DQ008832

Qiu 90031; OD/FBQ/YQ; DQ008783

Chase 219, NCU; OD/FBQ/ YQ; DQ008784

Qiu 97112; OD/FBQ/YQ; DQ008813

Qiu 94140; OD/FBQ/YQ;

DQ008814

Qiu 91019; OD/FBQ/YQ; DQ008796

Kuhlman sn, IND, Qiu 96018*; OD/FBQ/YQ;

DQ008799

Qiu 91018; OD/FBQ/YQ; DQ008800

Chase 1211, K; OD/FBQ/YQ; DQ008797

Qiu 94063; OD/FBQ/YQ; DQ008807
Fuse and Tamura 2000; AB040154

Fuse and Tamura 2000; AB040155

Fuse and Tamura 2000; AB040179

Hilu et al. 2003; B. Hall sn, BONN AF543721 (TB)

K.W. Hilu, K. Müller, and T. Borsch, unpublished manuscript; Borsch 3460, BONN AF543722 (KH)

Borsch \& Löhne 3555, BONN Chase 219, NCU; OD/YQ; AY437817

DQ008635

Hilu et al. 2003; Borsch 3457, Qiu 97112; OD/YQ; BONN AF543744 (KH) DQ008652

Hilu et al. 2003; Borsch 3408 BONN AF542575

Zanis et al. 2003; AY095473

Zanis et al. 2003; AY095450

Hilu et al. 2003; Borsch 3257, FR AF543724 (TB)

\section{Hilu et al. 2003; Huber sn,} BONN AF542571 (TB)

Murata et al. 2001; AB060736 Qiu 91018; OD/YQ; DQ008644

Kuhlman sn, IND, Qiu 96018*; OD/YQ;

DQ008643

Murata et al. 2001; AB060738 DQ008642

Qiu 94063; OD/YQ; DQ008646
J. Yamashita et al., unpublished data; AB029805 
Atherospermataceae:

Atherosperma moschatum Labill.

Daphnandra micrantha (Tul.) Benth.

Doryphora sassafras Endl.

Austrobaileyaceae:

Austrobaileya scandens C. T. White

Berberidaceae:

Mahonia bealei (Fortune) Carr.

Mahonia japonica

Podophyllum peltatum $\mathrm{L}$.

Buxaceae:

Buxus sempervirens $\mathrm{L}$.

Buxus sp.

Pachysandra procumbens Michx.

Pachysandra terminalis Sieb. \& Zucc.

Cabombaceae:

Brasenia schreberi J. Gmelin

Cabomba sp.

Cabomba caroliniana A. Gray

Calycanthaceae:

Calycanthus floridus L.

Calycanthus occidentalis Hook. \& Arn. Chimonanthus praecox (L.) Link

Canellaceae:

Canella winterana (L.) Gaertn.

Cinnamodendron ekmanii Sleum.
Qiu 92007; JL/LL/YQ;

DQ008679

Whalen 132, NSW; JL/LL/YQ;

DQ008680

Qiu 98109; JL/LL/YQ;

DQ008681

Parkinson et al. 1999; AF193988

Qiu 74; JL/LL/YQ; DQ008682

Qiu 92003; JL/LL/YQ;

DQ008683

Parkinson et al. 1999; AF193996

Chase 207, NCU; JL/LL/YQ

$$
\text { DQ008684 }
$$

\section{Qiu 91031; JL/LL/YQ; \\ DQ008685 \\ Parkinson et al. 1999; \\ AF193982;}

Parkinson et al. 1999; AF193989

$$
\begin{aligned}
& \text { Qiu 94155; OD/FBQ/YQ; } \\
& \text { DQ008780 }
\end{aligned}
$$

Qiu 9; JL/LL/YQ; DQ008686

Qiu 9; OD/FBQ/YQ;

Qiu 90017; JL/LL/YQ;

DQ008687

Qiu 90017; OD/FBQ/YQ;

Zanoni \& Jimenez 47067; JL/LL/YQ; DQ008688

Zanoni \& Jimenez 47067;
Qiu 92007; Kew; AJ966790

Whalen 132, NSW; Kew;

AJ966791

Hilu et al. 2003; Borsch 3409, BONN AF542568 (TB)

Hilu et al. 2003; Borsch 3464 BONN AF543726 (TB)

Qiu 74; OD/YQ; DQ008613

Hilu et al. 2003; Borsch 3405 , BONN AF542585 (TB)

Hilu et al. 2003; Borsch 3393, Qiu 92003; OD/YQ;

BONN AF542586 (TB)

DQ008614

Qiu 97057; Kew; AJ966792

S. Kim et al., unpublished data; AF389243

Qiu sn, Z (Qiu L99028*); OD/YQ; DQ008607

Hilu et al. 2003; Borsch 3407 , BONN AF542581 (KH)

Les et al. 1999; AF092973

Qiu 91031; LL/YQ; DQ008661

Les et al. 1999; AF108719

Soltis et al. 2003; AF479239

K.W. Hilu, K. Müller, and T. Borsch, unpublished manuscript; Borsch 3455 BONN AF543730 (KH)

Hilu et al. 2003; Borsch 3396, BONN AF542569 (TB)

K.W. Hilu, K. Müller, and T. Borsch, unpublished manuscript; Borsch 3466

BONN AF543731 (TB) OD/FBQ/YQ; DQ008805
Zanoni \& Jimenez 47067;

Kew; AJ966793
DQ008628

132, NSW; OD/YQ;

DQ008629

DQ008630

Zanis et al. 2003; AY095452
DQ008781 DQ008804

DQ008830

Qiu 97027; OD/FBQ/YQ;
Zanis et al. 2003; AY095455

Zanoni \& Jimenez 47067; MZ/DES/PSS AY095458 
Table 1

(Continued)

Family and species

mt-LSU rDNA

cp-matK

nu- $26 S$ rDNA

Ceratophyllaceae:

Ceratophyllum demersum $\mathrm{L}$.

Ceratophyllum submersum $\mathrm{L}$.

Chloranthaceae:

Ascarina rubricaulis Solms

Chloranthus brachystachys $\mathrm{Bl}$.

Chloranthus multistachys Pei

Hedyosmum arborescens Sw.

Hedyosmum bonplandianum $\mathrm{L}$.

Sarcandra chloranthoides Gardner

Cycadaceae:

Cycas revoluta Thunb.

Cycas panzhihuaensis

Cyclanthaceae:

Carludovica palmata Ruiz \& Pavon

Degeneriaceae:

Degeneria vitensis

Didymelaceae:

Didymeles perrieri Olivier

Dioscoreaceae:

Dioscorea sp.

Dioscorea alata

Eupomatiaceae:

Eupomatia bennettii F. Muell.

Eupteleaceae:

Euptelea polyandra Sieb. \& Zucc.

Fumariaceae:

Dicentra sp.

Dicentra exima Torrey
Parks sn, IND, Qiu 95003*; $\quad$ Parks sn, IND, Qiu 95003*; JL/LL/YQ; DQ008689

Qiu 98088; JL/LL/YQ;

DQ008690

Thien 500, NO; JL/LL/YQ;

DQ008691

K. Wurdack 92-0010, NCU; JL/LL/YQ; DQ008692 Chase 338, NCU; JL/LL/YQ; DQ008693

Parkinson et al. 1999; AF193992

Chaw et al. 2000; AB029356

Qiu 97021; JL/LL/YQ; DQ008694

JM Miller 1189-63; JL/LL/YQ; DQ008695

Andrianantoanina 387, MO; JL/LL/YQ; DQ008696

Qiu 94044; JL/LL/YQ; DQ008697

Qiu 90022; JL/LL/YQ; DQ008698

Parks sn, IND, Qiu 95098*; JL/LL/YQ; DQ008699

Qiu 95026; JL/LL/YQ; DQ008700
OD/FBQ/YQ; DQ008766

Qiu 98088; OD/FBQ/YQ;

$$
\text { DQ008767 }
$$

Thien 500, NO; OD/FBQ/YQ; DQ008821

Thien 500, NO Kew;

BONN AF543733 (KH)

K. Wurdack 92-0010, NCU; D/FBQ/YQ; DQ008819

Chase 338, NCU; OD/FBQ/ YQ; DQ008822

Qiu 92002; OD/FBQ/YQ; DQ008820

Qiu 94051; OD/FBQ/YQ; DQ008840

Qiu 97021; OD/FBQ/YQ; DQ008809

JM Miller 1189-63; OD/FBQ/ YQ; DQ008787

Andrianantoanina 387, MO; OD/FBQ/YQ; DQ008744

Qiu 94044; OD/FBQ/YQ; DQ008806

Qiu 90022; OD/FBQ/YQ; DQ008785

Parks sn, IND, Qiu 95098*; OD/FBQ/YQ; DQ008763

Qiu 95026; OD/FBQ/YQ; DQ008764

Parks sn, IND, Qiu 95098*;
Parks sn, IND, Qiu 95003*; Kew; AJ966794

Qiu 98088; Kew; AJ581400

AJ966795

Hilu et al. 2003; Borsch 3467

Zanis et al. 2003; AY095456

Qiu 98088; OD/YQ; DQ008622

Thien 500, NO; OD/YQ; DQ008656

Zanis et al. 2003; AY095457

Chase 338, NCU; Kew; AJ581402

Qiu 92002; Kew; AJ966796

Zanis et al. 2003; AY095461

Qiu 92002; OD/YQ;

DQ008655

Qiu 94051; OD/YQ; DQ008667

Wang et al. 2000; AF143440

Hilu et al. 2003; Roth sn, BONN AF542578 (TB)

Qiu 97021; OD/YQ; DQ008648

JM Miller 1189-63; OD/YQ; DQ008637

$$
\text { data; AB055549 }
$$

Andrianantoanina 387, MO; Kew; AJ581406 OD/YQ; DQ008608

Fuse and Tamura 2000 AB040208

Qiu 90022; Kew; AJ966797

Qiu 90022; OD/YQ;

DQ008636 Kew; AJ581413

Zanis et al. 2003; AF389249

Qiu 95026; Kew; AJ966798 
Ginkgoaceae:

Ginkgo biloba L.

Gnetaceae:

Gnetum gnemon $\mathrm{L}$

Gyrocarpaceae:

Gyrocarpus americana Jacq.

Hernandiaceae

Hernandia nymphaeifolia (Presl) Kub.

Hernandia ovigera L.

Himantandraceae:

Galbulimima belgraveana (F. Muell.) Sprague

Idiospermaceae:

Idiospermum australiense (Diels) S.T. Blake

Illiciaceae:

Illicium floridanum Ellis

Juncaginaceae:

Triglochin maritima L.

Lactoridaceae:

Lactoris fernandeziana Phil.

Lardizabalaceae:

Akebia quinata Decne.

Lardizabala biternata Ruiz \& Pavon

Lauraceae:

Cinnamomum camphora (L.) T. Nees \& Eberm.

Cryptocarya alba (Molina) Looser

Cryptocarya meisneriana Frodin

Laurus nobilis $\mathrm{L}$.

Magnoliaceae:

Liriodendron chinense (Hemsl.) Sarg.

Liriodendron tulipifera $\mathrm{L}$.

Magnolia denudata Desr.

Magnolia grandiflora L.
Chaw et al. 2000; AB029355

Qiu 94015; OD/FBQ/YQ; DQ008838

Qiu 97141; JL/LL/YQ;

DQ008701

Chase 317, NCU; JL/LL/YQ; DQ008702

Qiu 01007; JL/LL/YQ;

DQ008703

Weston 929, NSW; JL/LL/YQ; DQ008704

Qiu 91042; JL/LL/YQ; DQ008705

Qiu 61; JL/LL/YQ; DQ008706

Qiu 97141; OD/FBQ/YQ; DQ008833

Chase 317, NCU; OD/FBQ/ YQ; DQ008770

Qiu 01007; OD/FBQ/YQ; DQ008771

Weston 929, NSW; OD/FBQ/ YQ; DQ008788

Qiu 91042; OD/FBQ/YQ; DQ008782

Qiu 61; OD/FBQ/YQ; DQ008825

Qiu 97106; JL/LL/YQ; DQ008707

Chase 1014, K; JL/LL/YQ; DQ008708

Qiu 91020; JL/LL/YQ; DQ008709

Qiu 97135; JL/LL/YQ;

DQ008710

Qiu 102; JL/LL/YQ; DQ008711

Qiu 98048; JL/LL/YQ; DQ008712

Parkinson et al. 1999 AF193990

Parkinson et al. 1999; AF193993

Qiu 97106; OD/FBQ/YQ; DQ008811 Chase 1014, K; OD/FBQ/YQ;
DQ008798

Qiu 91020; OD/FBQ/YQ; DQ008761

Qiu 97135; OD/FBQ/YQ DQ008760

Qiu 102; OD/FBQ/YQ; DQ008772

Qiu 98048; OD/FBQ/YQ DQ008774

Qiu 94209; OD/FBQ/YQ; DQ008773

Qiu 28; OD/FBQ/YQ; DQ008786
Hilu et al. 2003; Borsch 3469 BONN AF543736 (KH)

Hilu et al. 2003; Borsch $3470 \quad$ Kuzoff et al. 1998; AF036488 BONN AF542561 (KH)

Chase 317, NCU; Kew; AJ581417

Qiu 01007; Kew; AJ966799

Weston 929, NSW; Kew; AF465294

Qiu 91042; Kew; AJ581425

K.W. Hilu, K. Müller, and T. Borsch, unpublished manuscript; Borsch 3552, BONN AF543738 (TB)

Hilu et al. 2003 Borsch 3392 BONN AF542566 (TB)

L.W. Chatrou et al., unpublished data; AF465297

Hilu et al. 2003; Borsch 3412 BONN AF542587 (TB) Qiu 97135; TB/KH; AY437809

Qiu 102; Kew; AJ966800

Rohwer 2000; AJ247158

Qiu 94209; Kew; AJ966801

Qiu 98048; OD/YQ
DQ008627

Qiu 94209; OD/YQ;

DQ008626

Zanis et al. 2003; AY095464

Azuma et al. 1999; AB021016 
Table 1

(Continued)

\begin{tabular}{|c|c|c|c|c|}
\hline Family and species & mt-SSU rDNA & mt-LSU rDNA & cp-matK & nu- $26 S$ rDNA \\
\hline Magnolia tripetala $\mathrm{L}$. & & $\begin{array}{l}\text { Qiu 3; OD/FBQ/YQ; } \\
\text { DQ008741 }\end{array}$ & Azuma et al. 1999; AB021001 & \\
\hline \multicolumn{5}{|l|}{ Menispermaceae: } \\
\hline Cissampelos pareira $\mathrm{L}$. & $\begin{array}{l}\text { Chase 347, NCU; JL/LL/YQ; } \\
\text { DQ008713 }\end{array}$ & $\begin{array}{l}\text { Chase } 347, \mathrm{NCU} \text {; OD/FBQ/ } \\
\text { YQ; DQ008758 }\end{array}$ & $\begin{array}{l}\text { Chase 347, NCU; Kew; } \\
\text { AJ966802 }\end{array}$ & $\begin{array}{l}\text { Chase 347, NCU; OD/YQ; } \\
\text { DQ008616 }\end{array}$ \\
\hline Cocculus trilobus (Thunb.) DC & $\begin{array}{l}\text { Qiu 91016; JL/LL/YQ; } \\
\text { DQ008714 }\end{array}$ & $\begin{array}{l}\text { Qiu 91016; OD/FBQ/YQ; } \\
\text { DQ008759 }\end{array}$ & & $\begin{array}{l}\text { Qiu 91016; OD/YQ; } \\
\text { DQ008617 }\end{array}$ \\
\hline Cocculus laurifolius DC. & & & $\begin{array}{l}\text { Hilu et al. 2003; Borsch } 3406 \\
\text { BONN AF542588 (TB) }\end{array}$ & \\
\hline \multicolumn{5}{|l|}{ Monimiaceae: } \\
\hline Hedycarya arborea J.R. \& G. Forst. & $\begin{array}{l}\text { Qiu 90028; JL/LL/YQ; } \\
\text { DQ008716 }\end{array}$ & $\begin{array}{l}\text { Qiu 90028; OD/FBQ/YQ; } \\
\text { DQ008769 }\end{array}$ & Qiu 90028; Kew; AJ581436 & $\begin{array}{l}\text { Qiu 90028; LL/YQ; } \\
\text { DQ008623 }\end{array}$ \\
\hline Hortonia floribunda Wight ex Arn. & $\begin{array}{l}\text { Qiu 02002*; JL/LL/YQ; } \\
\text { DQ008717 }\end{array}$ & $\begin{array}{l}\text { Qiu 02002*; OD/FBQ/YQ; } \\
\text { DQ008778 }\end{array}$ & Qiu 02002*; TB; AY437811 & Zanis et al. 2003; AF264143 \\
\hline Peumus boldus Molina & $\begin{array}{l}\text { Edinburgh BG 19870707; } \\
\text { JL/LL/YQ; DQ008715 }\end{array}$ & $\begin{array}{l}\text { Edinburgh BG 19870707; } \\
\text { OD/FBQ/YQ; DQ008768 }\end{array}$ & Rohwer 2000; AJ247183 & Zanis et al. 2003; AY095466 \\
\hline \multicolumn{5}{|l|}{ Myristicaceae: } \\
\hline Mauloutchia chapelieri Warb. & $\begin{array}{l}\text { Schatz 3847A, MO; JL/LL/YQ; } \\
\text { DQ008719 }\end{array}$ & $\begin{array}{l}\text { Schatz 3847A, MO; OD/FBQ/ } \\
\quad \text { YQ; DQ008790 }\end{array}$ & $\begin{array}{l}\text { Schatz 3847A, MO; TB; } \\
\quad \text { AY437812 }\end{array}$ & $\begin{array}{l}\text { Schatz 3847A, MO; OD/YQ; } \\
\text { DQ008638 }\end{array}$ \\
\hline Myristica fragrans Houtt. & $\begin{array}{l}\text { Qiu 92014; JL/LL/YQ; } \\
\text { DQ008718 }\end{array}$ & $\begin{array}{l}\text { Qiu 92014; OD/FBQ/YQ; } \\
\text { DQ008789 }\end{array}$ & Qiu 92014; Kew; AJ966803 & \\
\hline \multicolumn{5}{|l|}{ Nelumbonaceae: } \\
\hline Nelumbo lutea (Willd.) Pers. & & & & Zanis et al. 2003; AF389259 \\
\hline Nelumbo nucifera Gaertner & $\begin{array}{l}\text { Parkinson et al. 1999; } \\
\text { AF193983 }\end{array}$ & $\begin{array}{l}\text { Qiu 91028; OD/FBQ/YQ; } \\
\text { DQ008753 }\end{array}$ & $\begin{array}{l}\text { K.W. Hilu, K. Müller, and } \\
\text { T. Borsch, unpublished } \\
\text { manuscript; Borsch \& } \\
\text { Summers 3220, } \\
\text { FR AF543740 (TB) }\end{array}$ & \\
\hline \multicolumn{5}{|l|}{ Nymphaeaceae: } \\
\hline Nuphar sp. & $\begin{array}{l}\text { Parkinson et al. 1999; } \\
\quad \text { AF193981 }\end{array}$ & $\begin{array}{l}\text { Qiu M114; OD/FBQ/YQ; } \\
\text { DQ008829 }\end{array}$ & & $\begin{array}{l}\text { Qiu M114; LL/YQ; } \\
\text { DQ008660 }\end{array}$ \\
\hline Nuphar lutea L. & & & $\begin{array}{l}\text { Hilu et al. 2003; Borsch 3337, } \\
\text { FR AF543741 (TB) }\end{array}$ & \\
\hline Nymphaea sp. & Chaw et al. 2000; AF161091 & $\begin{array}{l}\text { Qiu 91029; OD/FBQ/YQ; } \\
\text { DQ008828 }\end{array}$ & & Zanis et al. 2003; AY095465 \\
\hline Nymphaea odorata Aiton & & & $\begin{array}{l}\text { Hilu et al. 2003; Borsch \& } \\
\text { Wilde } 3132 \text {, VPI \& BONN } \\
\text { AF543742 (TB) }\end{array}$ & \\
\hline \multicolumn{5}{|l|}{ Papaveraceae: } \\
\hline Sanguinaria canadensis $\mathrm{L}$. & $\begin{array}{l}\text { Qiu 91032; JL/LL/YQ; } \\
\text { DQ008720 }\end{array}$ & $\begin{array}{l}\text { Qiu 91032; OD/FBQ/YQ; } \\
\text { DQ008765 }\end{array}$ & Qiu 91032; Kew; AJ966804 & $\begin{array}{l}\text { Qiu 91032; OD/YQ; } \\
\text { DQ008621 }\end{array}$ \\
\hline \multicolumn{5}{|l|}{ Pinaceae: } \\
\hline Pinus sp. & $\begin{array}{l}\text { Qiu 94013; JL/LL/YQ; } \\
\text { DQ008721 }\end{array}$ & $\begin{array}{l}\text { Qiu 94013; OD/FBQ/YQ; } \\
\text { DQ008835 }\end{array}$ & & \\
\hline Pinus douglasiana Martinez & & & $\begin{array}{l}\text { L. G. Geada et al., unpublished } \\
\text { data; AB063520 }\end{array}$ & \\
\hline
\end{tabular}




\section{Piperaceae:}

Peperomia graveolens Rauh \& Barthlott

Peperomia obtusifolic

A. Dietr.

Piper betle $\mathrm{L}$.

Piper crocatum R. \& P.

Platanaceae:

Platanus occidentalis $\mathrm{L}$.

Podocarpaceae:

Podocarpus costalis Presl

Podocarpus macrophyllus (Thunb.) Sweet

Potamogetonaceae:

Potamogeton berchtoldii Fieber

Potamogeton distinctus Arth. Benn.

Proteaceae:

Grevillea banksii $\mathrm{R}$. Br.

Grevillea robusta Cunn. \& R. Br.

Persoonia katerae P. Weston \& L. Johnson

Petrophile canescens Cunn. ex R. Br.

Ranunculaceae:

Ranunculus sp.

Ranunculus ficaria L.

Ranunculus keniensis Milne-Redhead \& Turrill Xanthorhiza simplicissima Marshall

Sabiaceae:

Sabia sp.

Sabia swinhoei Hemsl.

Meliosma squamulata Hance

Meliosma veitchiorum Hemsl.

Sargentodoxaceae:

Sargentodoxa cuneata (Oliv.) Rehder \& Wilson
Qiu 94135;

JL/LL/YQ; DQ008722

Chaw et al. 2000; AF161088

Chaw et al. 2000; AF161090

Chaw et al. 2000; AF029369

Qiu 96063; JL/LL/YQ;

DQ008723

Parkinson et al. 1999; AF193995

Weston 1120, NSW; JL/LL/YQ DQ008724

Qiu 98018; JL/LL/YQ;

DQ008725

Chaw et al. 2000; AF161093

Qiu 91030; JL/LL/YQ;

DQ008726

Qiu 91025; JL/LL/YQ; DQ008727

Qiu 94135;

OD/FBQ/YQ; DQ008794

Qiu 91048; OD/FBQ/YQ;

DQ008795

Qiu 94152; OD/FBQ/YQ; DQ008752

Qiu 95006; OD/FBQ/YQ; DQ008837

Qiu 96063; OD/FBQ/YQ; DQ008810

Qiu 94087; OD/FBQ/YQ; DQ008751

Weston 1120, NSW; OD/FBQ/ YQ; DQ008750 Qiu 98018; OD/FBQ/YQ; DQ008749

Qiu 95024; OD/FBQ/YQ; DQ008756

\section{Qiu 91030; OD/FBQ/YQ;} DQ008757

Qiu 91025; OD/FBQ/YQ; DQ008747

B. Shih 3749, HAST; JL/LL/YQ; B. Shih 3749, HAST; OD/FBQ/ DQ008728 YQ; DQ008748

Pan 93001, NCU; JL/LL/YQ; DQ008729
Hilu et al. 2003; Prinsler s.n.

BONN AF542574 (TB)

Qiu 94135;

LL/YQ; DQ008641

Zanis et al. 2003; AY095467

Hilu et al. 2003; Slotta s.n., VPI AF543745 (KH)

K.W. Hilu et al., unpublished data; AF543747

Wang and Shu 2000; AF228111

DQ008664

Qiu 96063; OD/YQ; DQ008649

Tanaka et al. 1997; AB002581

Hilu et al. 2003; Borsch 3413 BONN AF542583 (TB)

Weston 1120, NSW; TB; AY437813

Qiu 98018; Kew; AJ966805

DQ008612

Weston 1120, NSW; OD/YQ DQ008611

Qiu 98018; OD/YQ;

DQ008610

Borsch 3554 BONN; TB; AY437814

Zanis et al. 2003; AF389269

Hilu et al. 2003; Borsch 3394 Qiu 91030; OD/YQ; BONN AF542567 (TB) DQ008615

Qiu 91025; Kew; AJ966806

Zanis et al. 2003; AF389272

B. Shih 3749, HAST; OD/YQ DQ008609

Chase 2989, K; Kew; AJ581449

Pan 93001, NCU; Kew;

AJ966807
Pan 93001, NCU; OD/YQ; DQ008620 


\section{Table 1}

(Continued)

\begin{tabular}{|c|c|c|c|c|}
\hline$\underline{\text { Family and species }}$ & mt-SSU rDNA & mt-LSU rDNA & cp-matK & nu- $26 S$ rDNA \\
\hline \multicolumn{5}{|l|}{ Saururaceae: } \\
\hline Anemopsis californica (Nutt.) Hook. \& Arn. & $\begin{array}{l}\text { Qiu 97116; JL/LL/YQ; } \\
\text { DQ008730 }\end{array}$ & $\begin{array}{l}\text { Qiu 97116; OD/FBQ/YQ; } \\
\text { DQ008791 }\end{array}$ & $\begin{array}{l}\text { Borsch } 3397 \text { BONN; TB; } \\
\quad \text { AY437810 }\end{array}$ & $\begin{array}{l}\text { Qiu 97116; OD/YQ; } \\
\text { DQ008639 }\end{array}$ \\
\hline Houttuynia cordata Thunb. & $\begin{array}{l}\text { Qiu 92016; JL/LL/YQ; } \\
\text { DQ008731 }\end{array}$ & $\begin{array}{l}\text { Qiu 92016; OD/FBQ/YQ; } \\
\text { DQ008793 }\end{array}$ & $\begin{array}{l}\text { K.W. Hilu, K. Müller, and } \\
\text { T. Borsch, unpublished } \\
\text { manuscript; Borsch } 3481 \\
\text { BONN AF543737 (TB) }\end{array}$ & $\begin{array}{l}\text { Qiu 92016; OD/YQ; } \\
\text { DQ008640 }\end{array}$ \\
\hline Saururus cernuus L. & $\begin{array}{l}\text { Qiu 97098*; JL/LL/YQ; } \\
\text { DQ008732 }\end{array}$ & $\begin{array}{l}\text { Qiu } 97098 * \text {; OD/FBQ/YQ; } \\
\text { DQ008792 }\end{array}$ & $\begin{array}{l}\text { K.W. Hilu, K. Müller, and } \\
\text { T. Borsch, unpublished } \\
\text { manuscript; Borsch \& } \\
\text { Wilde } 3108 \text {, VPI \& FR } \\
\text { AF543749 (TB) }\end{array}$ & Zanis et al. 2003; AY095468 \\
\hline \multicolumn{5}{|l|}{ Schisandraceae: } \\
\hline Kadsura japonica (L.) Dunal & $\begin{array}{l}\text { Parkinson et al. 1999; } \\
\text { AF193985 }\end{array}$ & $\begin{array}{c}\text { Parks sn, IND, Qiu 94159*; } \\
\text { OD/FBQ/YQ; DQ008823 }\end{array}$ & $\begin{array}{l}\text { Hilu et al. 2003; Borsch 3411, } \\
\text { BONN AF542565 (KH) }\end{array}$ & $\begin{array}{l}\text { Parks sn, IND, Qiu 94159*; } \\
\text { OD/YQ; DQ008657 }\end{array}$ \\
\hline Schisandra rubriflora & & & $\begin{array}{l}\text { K.W. Hilu, K. Müller, and } \\
\text { T. Borsch, unpublished } \\
\text { manuscript; Borsch } 3477 \text {; } \\
\text { BONN AF543750 }(\mathrm{KH})\end{array}$ & \\
\hline Schisandra sphenanthera Rehd. \& Wils. & $\begin{array}{l}\text { Parkinson et al. 1999; } \\
\text { AF193984 }\end{array}$ & $\begin{array}{l}\text { Parks sn, IND, Qiu 94165*; } \\
\text { OD/FBQ/YQ; DQ008824 }\end{array}$ & & $\begin{array}{l}\text { Parks sn, IND, Qiu 94165*; } \\
\text { OD/YQ; DQ008658 }\end{array}$ \\
\hline \multicolumn{5}{|l|}{ Siparunaceae: } \\
\hline Siparuna decipiens (Tul.) A. DC. & $\begin{array}{l}\text { Sothers 911, MO; JL/LL/YQ; } \\
\text { DQ008733 }\end{array}$ & $\begin{array}{l}\text { Sothers 911, MO; OD/FBQ/ } \\
\text { YQ; DQ008779 }\end{array}$ & $\begin{array}{l}\text { Sothers 911, MO; Kew; } \\
\text { AJ966808 }\end{array}$ & $\begin{array}{l}\text { Sothers 911, MO; OD/YQ; } \\
\text { DQ008631 }\end{array}$ \\
\hline \multicolumn{5}{|l|}{ Stemonaceae: } \\
\hline Croomia pauciflora Miq. & $\begin{array}{l}\text { Qiu 97096*; JL/LL/YQ; } \\
\text { DQ008734 }\end{array}$ & $\begin{array}{l}\text { Qiu 97096*; OD/FBQ/YQ; } \\
\text { DQ008808 }\end{array}$ & Qiu 97096*; TB; AY437815 & $\begin{array}{l}\text { Qiu 97096*; OD/YQ; } \\
\text { DQ008647 }\end{array}$ \\
\hline \multicolumn{5}{|l|}{ Taxodiaceae: } \\
\hline Metasequoia glyptostroboides $\mathrm{Hu} \&$ Cheng & & $\begin{array}{l}\text { Qiu 95084; OD/FBQ/YQ; } \\
\text { DQ008836 }\end{array}$ & Gadek et al. 2000; AF152203 & $\begin{array}{l}\text { Qiu 95084; OD/YQ; } \\
\text { DQ008663 }\end{array}$ \\
\hline \multicolumn{5}{|l|}{ Tetracentraceae: } \\
\hline Tetracentron sinensis Oliv. & $\begin{array}{l}\text { Parkinson et al. 1999; } \\
\text { AF193998 }\end{array}$ & $\begin{array}{l}\text { Qiu 90009; OD/FBQ/YQ; } \\
\text { DQ008745 }\end{array}$ & Fishbein et al. 2001; AF274633 & Fishbein et al. 2001; AF274670 \\
\hline \multicolumn{5}{|l|}{ Tofieldiaceae: } \\
\hline Pleea tenufolia Michaux & $\begin{array}{l}\text { Qiu 96128*; JL/LL/YQ; } \\
\text { DQ008735 }\end{array}$ & $\begin{array}{l}\text { Qiu 96128*; OD/FBQ/YQ; } \\
\text { DQ008815 }\end{array}$ & $\begin{array}{l}\text { L.W. Chatrou et al., } \\
\text { unpublished data; AF465301 }\end{array}$ & Zanis et al. 2003; AY095472 \\
\hline Tofieldia calyculata (L.) Wahlenb. & $\begin{array}{l}\text { Qiu 97041; JL/LL/YQ; } \\
\text { DQ008736 }\end{array}$ & $\begin{array}{l}\text { Qiu 97041; OD/FBQ/YQ; } \\
\text { DQ008816 }\end{array}$ & & $\begin{array}{l}\text { Qiu 97041; OD/YQ; } \\
\text { DQ008653 }\end{array}$ \\
\hline Tofieldia racemosa & & & $\begin{array}{l}\text { Fuse and Tamura 2000; } \\
\text { AB040160 }\end{array}$ & \\
\hline \multicolumn{5}{|l|}{ Trimeniaceae: } \\
\hline Trimenia moorei W.R. Philipson & $\begin{array}{l}\text { ANBG 701680; JL/LL/YQ; } \\
\text { DQ008737 }\end{array}$ & $\begin{array}{l}\text { ANBG 701680; OD/FBQ/YQ; } \\
\text { DQ008826 }\end{array}$ & $\begin{array}{l}\text { ANBG 701680; Kew; } \\
\text { AJ966809 }\end{array}$ & Zanis et al. 2003; AY095470 \\
\hline \multicolumn{5}{|l|}{ Trochodendraceae: } \\
\hline Trochodendron aralioides Sieb. \& Zucc. & Qiu 49; JL/LL/YQ; DQ008738 & $\begin{array}{l}\text { Qiu 49; OD/FBQ/YQ; } \\
\text { DQ008746 }\end{array}$ & Fishbein et al. 2001; AF274634 & Fishbein et al. 2001; AF274671 \\
\hline
\end{tabular}


Welwitschiaceae:

Welwitschia mirabilis Hook. $\mathrm{f}$

Chaw et al. 2000; AF161083

Drimys winteri J.R. \& G. Forster

Takbtajania perrieri M. Baranova \& J. Lero

Tasmannia insipida DC

\section{Zamiaceae:}

Zamia floridana A. DC.

Zamia furfuracea Aiton AF197162

Rabenantoandro 219, MO; JL/LL/YQ; DQ008740

Qiu 90032; JL/LL/YQ;

DQ008739

Chaw et al. 2000; AF029357
Parkinson et al. 1999;
Qiu M44; OD/FBQ/YQ; DQ008834
Qiu 90016; OD/FBQ/YQ; DQ008801

Rabenantoandro 219, MO; OD/FBQ/YQ; DQ008803

iu 90032; OD/FBQ/YQ; DQ008802
Hilu et al. 2003; Borsch 3410 BONN AF542562 (TB)

Borsch 3479 , BONN; TB AY437816

Rakotomalaza et al. 1342

MO; Kew; AJ581455

Qiu 90032; Kew; AJ966810

Qiu 95035; OD/FBQ/YQ; DQ008839
Qiu M44. OD/YQ. DQ008662

Kuzoff et al. 1998; AF036491

Rabenantoandro 219, MO

$\mathrm{OD} / \mathrm{YQ} ; \mathrm{DQ} 008645$

Zanis et al. 2003; AY095469

Qiu 95035; OD/YQ;

DQ008666

S. Zhang et al., unpublished

data; AF410170

Note. Vouchers with numbers between Qiu 1 and Qiu 93999 are deposited in NCU, Qiu 94001-Qiu 97999 in IND, Qiu 98001-Qiu 99999 in Z, and Qiu 00001-Qiu 02999 in MICH. Vouchers by collectors other than Qiu are indicated with the herbaria where they have been deposited. Sequence contributors: DES, Douglas E. Soltis; FBQ, Fabiana Bernasconi-Quadroni; KH, Khidir Hilu; JL, Jungho Lee; LL, Libo Li; MZ, Michael Zanis; OD, Olena Dombrovska; PSS, Pamela S. Soltis; TB, Thomas Borsch; YQ, Yin-Long Qiu. Numbers labeled with asterisks are DNA numbers (no voucher or a voucher by someone without a number). 
Table 2

Information on New Sequences and Replacements for the Five Genes Used by Qiu et al. (2000) and Correction of Errors in Table A1 of Qiu et al. (2000)

\begin{tabular}{|c|c|c|c|c|c|}
\hline Family and species & mt-atp1 & $\mathrm{mt}-\mathrm{mat} R$ & cp-atpB & cp-rbcL & nu-18S rDNA \\
\hline \multicolumn{6}{|l|}{ Acoraceae: } \\
\hline Acorus calamus L. & & $\begin{array}{l}\text { Qiu 94052; OD/YQ; } \\
\text { DQ007422 }\end{array}$ & & & \\
\hline Acorus gramineus Soland. & & $\begin{array}{l}\text { Qiu 97131; OD/YQ; } \\
\text { DQ007423 }\end{array}$ & & & \\
\hline \multicolumn{6}{|l|}{ Alismataceae: } \\
\hline Alisma plantago-aquatica $\mathrm{L}$. & & & $\begin{array}{l}\text { Qiu 96177; LL/YQ; } \\
\text { DQ007417 }\end{array}$ & & \\
\hline \multicolumn{6}{|l|}{ Amborellaceae: } \\
\hline Amborella trichopoda Baill. & $\begin{array}{l}\text { B. Hall sn, IND, Qiu } \\
\text { 97123*; JL/YQ; } \\
\text { DQ007412 }\end{array}$ & & & & \\
\hline \multicolumn{6}{|l|}{ Annonaceae: } \\
\hline Cananga odorata (Lam.) Hook. f. \& Thomson & & & $\begin{array}{l}\text { Chase 219, NCU; } \\
\text { LL/YQ; DQ007418 }\end{array}$ & & \\
\hline \multicolumn{6}{|l|}{ Aristolochiaceae: } \\
\hline Asarum canadense $\mathrm{L}$. & & & $\begin{array}{l}\text { Hoot et al. 1999; } \\
\text { U86383 }\end{array}$ & & \\
\hline Thottea tomentosa Ding Hou & & & & & $\begin{array}{l}\text { Chase 1211, K; LL/YQ; } \\
\text { DQ007406 }\end{array}$ \\
\hline \multicolumn{6}{|l|}{ Atherospermaceae: } \\
\hline Daphnandra repandula F. Muell. & & & & $\begin{array}{l}\text { Renner et al. 1998; } \\
\quad \text { AF052195 }\end{array}$ & \\
\hline Doryphora aromatica (F.M. Bailey) L.S. Sm. & & & & $\begin{array}{l}\text { E. E. M. Ablett et al., } \\
\text { unpublished data; } \\
\text { L77211 }\end{array}$ & \\
\hline \multicolumn{6}{|l|}{ Cabombaceae: } \\
\hline Brasenia schreberi J. Gmelin & & & & Les et al. 1991; M77031 & \\
\hline Cabomba caroliniana A. Gray & & & $\begin{array}{l}\text { Graham and Olmstead } \\
2000 b ; \text { AF187058 }\end{array}$ & Les et al. 1991; M77027 & \\
\hline \multicolumn{6}{|l|}{ Calycanthaceae: } \\
\hline Chimonanthus praecox (L.) Link & & & & & $\begin{array}{l}\text { Soltis et al. 2000; } \\
\text { AF503352 }\end{array}$ \\
\hline \multicolumn{6}{|l|}{ Canellaceae: } \\
\hline Cinnamodendron ekmanii Sleum. & & & & $\begin{array}{l}\text { Zanoni \& Jimenez } \\
\text { 47067; LL/YQ; } \\
\text { DQ007428 }\end{array}$ & \\
\hline \multicolumn{6}{|l|}{ Ceratophyllaceae: } \\
\hline Ceratophyllum demersum $\mathrm{L}$. & & & $\begin{array}{l}\text { Savolainen et al. 2000; } \\
\text { AJ235430 }\end{array}$ & & \\
\hline \multicolumn{6}{|l|}{ Chloranthaceae: } \\
\hline Ascarina rubricaulis Solms & $\begin{array}{l}\text { Thien 500, NO; } \\
\text { JL/FBQ/YQ; } \\
\text { AF197667 }\end{array}$ & $\begin{array}{l}\text { Thien 500, NO; } \\
\text { FBQ/JL/YQ; } \\
\text { AF197755 }\end{array}$ & & $\begin{array}{l}\text { Thien 500, NO; } \\
\text { ZC/JL/YQ; } \\
\text { AF197592 }\end{array}$ & \\
\hline
\end{tabular}


Hedyosmum arborescens $\mathrm{Sw}$

Cycadaceae:

Cycas revoluta Thunb.

Degeneriaceae:

Degeneria vitensis

Didymelaceae:

Didymeles perrieri Olivier

Dioscoreaceae

Eupomatiaceae:

Eupomatia bennettii F. Muell.

Eupteleaceae:

Euptelea polyandra Sieb. \& Zucc.

Ginkgoaceae:

Ginkgo biloba L.

Gnetaceae:

Gnetum gnemon L.

Gomortegaceae:

Gomortega keule (Molina) I.M. Johnson

Gyrocarpaceae:

Gyrocarpus americana Jacq.

Hernandiaceae:

Hernandia ovigera $\mathrm{L}$.

Himantandraceae:

Galbulimima belgraveana (F. Muell.) Sprague

Juncaginaceae:

Triglochin maritima L.

Lauraceae:

Cryptocarya obovata

\section{Monimiaceae:}

Peumus boldus Molina
Savolainen et al. 2000; AJ235491

Pryer et al. 2001;

AF313558

$\begin{array}{cc}\text { JM Miller 1189-63; } & \text { JM Miller 1189-63; } \\ \text { JL/FBQ/YQ; } & \text { FBQ/JL/YQ; } \\ \text { AF293752 } & \text { AF197771 }\end{array}$

Savolainen et al. 2000; AJ235451

Qiu et al. 1993; L12643

Soltis et al. 2000; AF206898

Hoot et al. 1999; AF094541

Caddick et al. 2002; AF308014

Soltis et al. 1997; AF469771

Hoot et al. 1999; U86384

Soltis et al. 1997; L75831

Savolainen et al. 2000; AJ235481

Graham and Olmstead 2000b; AF187060

Soltis et al. 2000; AF209593

Ueda et al. 1997; AF206773

Chase 317, NCU; $\mathrm{JL} / \mathrm{FBQ} / \mathrm{YQ}$ AF197701

Qiu 96255*; JL/YQ; DQ007413

Chase 317, NCU; FBQ/ JL/YQ; AF197805

AJ235487

Qiu et al. 1993; L12647 Soltis et al. 2000; AF206923

\section{Qiu 01007; LL/YQ;} DQ007424

\section{Qiu 01007; LL/YQ; DQ007419}

Qiu et al. 1993; L12650

Qiu 96255*; FBQ/YQ;

DQ007407
Savolainen et al. 2000; AJ235478
Les et al. 1997; U80714

\section{P. G. Martin and}

J. Dowd, unpublished data; L28950

Soltis et al. 2000; AF206807 
Table 2

(Continued)

\begin{tabular}{|c|c|c|c|c|c|}
\hline Family and species & mt-atp1 & mt-matR & сp-atpB & сp-rbcL & nu-18S rDNA \\
\hline $\begin{array}{l}\text { Hortonia floribunda Wight } \\
\text { ex Arn. }\end{array}$ & $\begin{array}{l}\text { Qiu M166; JL/YQ; } \\
\text { DQ007414 }\end{array}$ & & $\begin{array}{l}\text { Qiu 02002*; LL/YQ; } \\
\text { DQ007420 }\end{array}$ & $\begin{array}{l}\text { Renner 1998; } \\
\text { AF040663 }\end{array}$ & $\begin{array}{l}\text { Qiu 02002*; LL/YQ; } \\
\text { DQ007408 }\end{array}$ \\
\hline $\begin{array}{l}\text { Myristicaceae: } \\
\text { Mauloutchia chapelieri Warb. }\end{array}$ & & & & & $\begin{array}{l}\text { Schatz3847A, MO; } \\
\text { LL/YQ; DQ007409 }\end{array}$ \\
\hline $\begin{array}{l}\text { Nymphaeaceae: } \\
\text { Nuphar variegata Durand } \\
\text { Nymphaea odorata Aiton }\end{array}$ & & & & $\begin{array}{l}\text { Les et al. 1991; M77029 } \\
\text { Les et al. 1991; M77034 }\end{array}$ & \\
\hline $\begin{array}{l}\text { Piperaceae: } \\
\quad \text { Peperomia obtusifolia A. Dietr. }\end{array}$ & & & $\begin{array}{l}\text { Savolainen et al. 2000; } \\
\text { AJ235556 }\end{array}$ & & \\
\hline $\begin{array}{l}\text { Podocarpaceae: } \\
\text { Podocarpus costalis Presl }\end{array}$ & & & & & $\begin{array}{l}\text { Chaw et al. 1997; } \\
\text { D38473 }\end{array}$ \\
\hline Podocarpus macrophyllus (Thunb.) Sweet & & $\begin{array}{l}\text { Qiu 95006; FBQ/YQ; } \\
\text { DQ007425 }\end{array}$ & & & \\
\hline $\begin{array}{l}\text { Potamogetonaceae: } \\
\quad \text { Potamogeton berchtoldii Fieber }\end{array}$ & & & $\begin{array}{l}\text { Qiu 96063; FBQ/JL/ } \\
\text { YQ; AF197600 }\end{array}$ & & $\begin{array}{l}\text { Qiu 96063; LL/YQ; } \\
\text { DQ007410 }\end{array}$ \\
\hline $\begin{array}{l}\text { Proteaceae: } \\
\text { Persoonia lanceolata }\end{array}$ & & & & $\begin{array}{l}\text { G. M. Plunkett et al., } \\
\text { unpublished data; } \\
\text { U79178 }\end{array}$ & \\
\hline Petrophile circinata & & & $\begin{array}{l}\text { Hoot and Douglas } \\
\text { 1998; AF060401 }\end{array}$ & & \\
\hline $\begin{array}{l}\text { Sabiaceae: } \\
\text { Meliosma squamulata Hance }\end{array}$ & & $\begin{array}{l}\text { B. Shih 3749, HAST; } \\
\text { OD/YQ; DQ007426 }\end{array}$ & & & \\
\hline $\begin{array}{l}\text { Saururaceae: } \\
\text { Saururus cernuus L. }\end{array}$ & & & & & $\begin{array}{l}\text { Soltis et al. 1997; } \\
\text { U42805 }\end{array}$ \\
\hline $\begin{array}{l}\text { Schisandraceae: } \\
\text { Schisandra chinensis }\end{array}$ & & & & & $\begin{array}{l}\text { Soltis et al. 1997; } \\
\quad \text { L75842 }\end{array}$ \\
\hline $\begin{array}{l}\text { Siparunaceae: } \\
\quad \text { Siparuna brasiliensis (Spreng.) A. DC. }\end{array}$ & & & $\begin{array}{l}\text { J. Lombardi 2714, MO; } \\
\text { LL/YQ; DQ007421 }\end{array}$ & & \\
\hline Siparuna decipiens A. DC. & & & & & $\begin{array}{l}\text { Sothers 911, MO; LL/ } \\
\text { YQ; DQ007411 }\end{array}$ \\
\hline $\begin{array}{l}\text { Stemonaceae: } \\
\text { Croomia pauciflora Miq. }\end{array}$ & & & & & $\begin{array}{l}\text { Caddick et al. 2002; } \\
\text { AF309408 }\end{array}$ \\
\hline Croomia japonica Miq. & & & $\begin{array}{l}\text { Caddick et al. 2002; } \\
\text { AF308039 }\end{array}$ & & \\
\hline
\end{tabular}


Tofieldiaceae:

Pleea tenufolia Michaux

Trimeniaceae:

Trimenia moorei W.R. Philipson

Welwitschiaceae:

Welwitschia mirabilis Hook. $\mathrm{f}$.

\section{Winteraceae:}

Takhtajania perrieri M. Baranova \& J. Leroy

Rabenantoandro 219,

$\mathrm{MO}$; LL/YQ;

Tasmannia insipida

DQ007416

Zamiaceae:

Zamia furfuracea Aiton

Zamia pumila $\mathrm{L}$.

ANBG 701680; JL/

YQ;DQ007415
Chase et al. 1993;

AJ131774

W. Graham et al.,

unpublished data; AF239795

Rabenantoandro 219,

MO; LL/YQ;

DQ007427

Soltis et al. 2000;

AF209683

Hoot et al. 1999; AF093424

Graham and Olmstead 2000a; AF188845

Note. New sequences are given in boldface. 


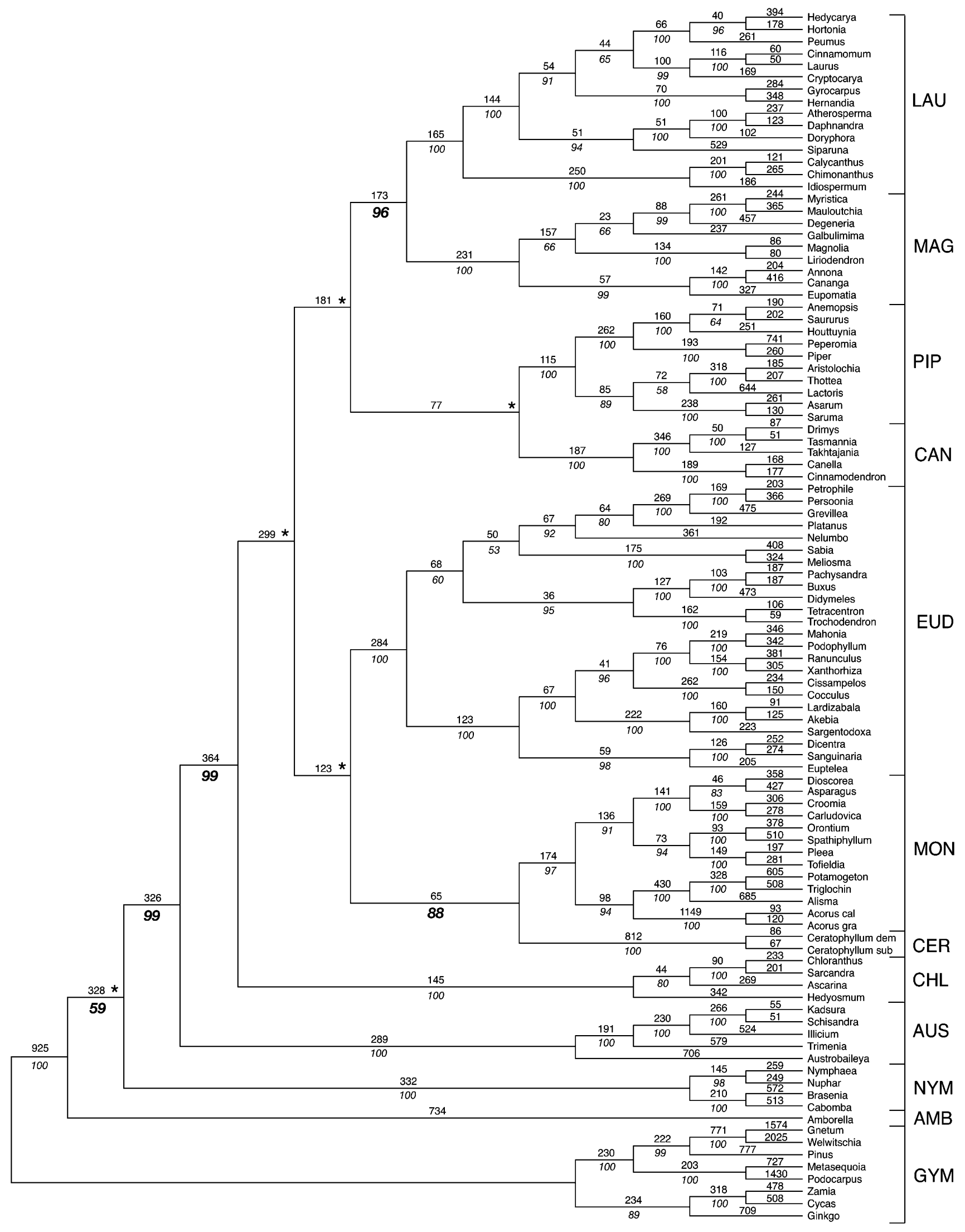


that are not present in the strict consensus of the two trees indicated by asterisks.

Because the tree topologies from the two parsimony searches are generally congruent, we describe them together. Amborella, Nymphaeaceae, and Austrobaileyales form successive sister lineages to the rest of the angiosperms, with generally strong bootstrap support (we regard bootstrap values of $50 \%-69 \%$ as weak, $70 \%-84 \%$ as moderate, and $85 \%$ and above as strong support; these cutoff values are designated for convenience of communication, but see Hillis and Bull 1993 for a discussion of phylogenetic implication of bootstrap values). However, the placement of Amborella as the sister to all other angiosperms is only weakly to moderately supported. Further, five strongly supported clades are recognized within the remaining angiosperms in the fiveprotein-gene analysis: monocots, Chloranthaceae, Ceratophyllum, magnoliids, and eudicots. In contrast, the monophyly of magnoliids did not receive support of $>50 \%$ in the nine-gene analysis. Ceratophyllum was moderately supported as the sister to eudicots in the five-protein-gene analysis but strongly supported as the sister to monocots in the nine-gene analysis. No other higher-level relationships among the basal angiosperms received bootstrap support above 50\%. Finally, within the magnoliids, the sister relationships between Magnoliales and Laurales, between Canellales and Piperales, and between these two larger clades are all strongly supported in the fiveprotein-gene analysis. In the nine-gene analysis, however, only the sister relationship between Magnoliales and Laurales received strong support. The bootstrap percentages of key nodes in the trees from analyses of nine genes, five protein genes, five protein genes plus $18 \mathrm{~S}$ rDNA, and five protein genes plus $18 \mathrm{~S}$ and $26 \mathrm{~S}$ rDNAs are presented in table 3.

The Bayesian analyses of the nine-gene and five-proteingene matrices produced similar topologies, with the sole difference being that monocots and eudicots switched position as the sister to magnoliids (fig. 3). There are two additional topological features that are seen in results of the Bayesian but not the parsimony analyses: Ceratophyllum is sister to Chloranthaceae ( $\mathrm{PP}=0.78$ and 0.92 in the nine-gene and five-protein-gene analyses, respectively), and Amborella is sister to Nymphaeaceae ( $\mathrm{PP}=1.00$ in both analyses). Otherwise, the topologies of the Bayesian and parsimony trees are similar.

The ML analyses of the nine-gene and five-protein-gene matrices also identified certain relationships that were recovered in the parsimony and Bayesian analyses, i.e., monophyly of magnoliids and placement of Amborella, Nymphaeales, and Austrobaileyales as successive sisters to all other extant angiosperms, but they differed on resolving relationships among Ceratophyllum, Chloranthaceae, magnoliids, monocots, and eudicots. Schematic presentations of the trees from both analyses and the bootstrap values are shown in figure 3 .

The parsimony bootstrap analyses of three genome-specific matrices produced similar topologies but with different support for various relationships among basal angiosperms (fig. 4). The positions of Amborella, Nymphaeaceae, and Austrobaileyales were supported by all three genome-specific analyses, with the plastid data set giving strong support and the mitochondrial and nuclear data sets providing only moderate to weak support, respectively. Chloranthaceae, Ceratophyllum, and eudicots were each recovered with strong support in all three single-genome analyses. Monocot monophyly was strongly supported by plastid data, moderately supported by mitochondrial data, and not supported by a bootstrap value $>50 \%$ by the nuclear data. The monophyly of magnoliids and relationships among the member clades (Magnoliales, Laurales, Canellales, and Piperales) received only weak support in the plastid genome analysis. The mitochondrial and nuclear data sets contained essentially no phylogenetic signal for recognizing this clade or for resolving relationships among its subclades, with the sole exception that the sister relationship between Magnoliales and Laurales is strongly supported by the mitochondrial data set.

In our examination of the nine-gene alignment, a total of 71 sites were identified that contain apparently synapomorphic substitutions that separate gymnosperms-AmborellaNymphaeaceae-Austrobaileyales and all other angiosperms (fig. 5). With these sites removed, both the shortest tree search and a bootstrapping analysis of the nine-gene matrix identified Ceratophyllum as the sister to all other angiosperms, with $55 \%$ bootstrap support. Amborella, Nymphaeaceae, and Austrobaileyales formed a weakly (63\%) supported clade as part of a trichotomy with monocots and a clade containing Chloranthaceae, magnoliids, and eudicots (data not shown). We also conducted a shortest tree search using the 71-site matrix (fig. 5), but because of limited information for resolving relationships among the shallow branches, the search did not finish because of the huge number of trees found and the corresponding computer memory shortage. However, in the trees recovered when the search was aborted, the angiosperms exclusive of Amborella, Nymphaeaceae, and Austrobaileyales did form a monophyletic group, with members of the latter three clades variously grouping with the gymnosperms (data not shown). These results confirm that our identification of the sites containing putatively synapomorphic substitutions was correct. The 71 sites are distributed throughout the entire length of each of the nine genes, with only 13 sites linked in five groups (fig. 5). They contain all six possible substitutional changes, with 38 sites exhibiting transitions between gymnospermsAmborella-Nymphaeaceae-Austrobaileyales and all other

Fig. 1 One of the six shortest trees found in the parsimony analysis of the nine-gene matrix. Numbers above branches are branch lengths (ACCTRAN optimization); those below in italics are bootstrap percentages (only those $>50 \%$ are shown; for branches related to Amborella, Nymphaeaceae, Austrobaileyales, Ceratophyllum, magnoliids, monocots, and eudicots, the bootstrap percentages are in boldface). The nodes labeled with asterisks are collapsed in the strict consensus of the six shortest trees. Abbreviations: GYM = gymnosperms; AMB $=$ Amborella; NYM = Nymphaeaceae; $\quad$ AUS = Austrobaileyales; $\quad$ CHL = Chloranthaceae; $\quad$ CER $=$ Ceratophyllum $; \quad M O N=$ monocots; $\quad$ EUD = eudicots; CAN = Canellales; PIP = Piperales $;$ MAG = Magnoliales; LAU = Laurales; Acorus cal = Acorus calamus $;$ Acorus gra $=$ Acorus gramineus $;$ Ceratophyllum dem $=$ Ceratophyllum demersum; Ceratophyllum sub = Ceratophyllum submersum. 


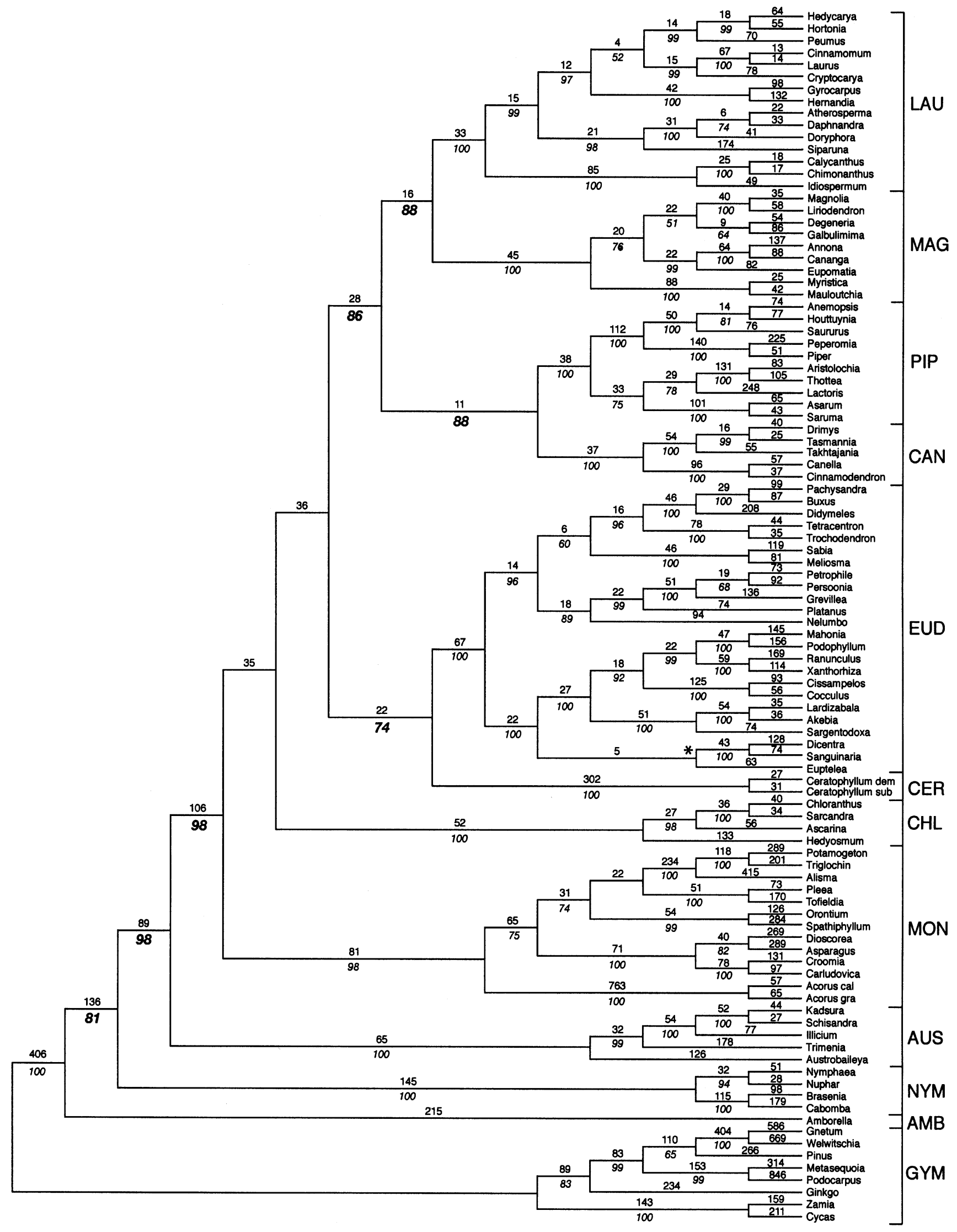


Table 3

Bootstrap (and Jackknife When Indicated) Percentages for a Subset of the Major Clades in the Tree Shown in Figures 1 and 2, and in Several Previous Studies

\begin{tabular}{|c|c|c|c|c|c|c|c|}
\hline Clade & $\begin{array}{l}\text { 3-gene (Soltis } \\
\text { et al. 2000) } \\
\text { (jack knife) }\end{array}$ & $\begin{array}{l}\text { 5-gene (Qiu } \\
\text { et al. 2000) }\end{array}$ & $\begin{array}{c}\text { 5-11 gene (Zanis } \\
\text { et al. 2002) }\end{array}$ & $\begin{array}{c}5 \text {-protein } \\
\text { (this study) }\end{array}$ & $\begin{array}{c}5 \text {-protein }+18 S \\
\text { (this study) }\end{array}$ & $\begin{array}{c}5 \text {-protein }+18 \mathrm{~S} \\
+26 \mathrm{~S} \text { (this } \\
\text { study) }\end{array}$ & $\begin{array}{l}\text { 9-gene } \\
\text { (this } \\
\text { study) } \\
\end{array}$ \\
\hline Amborella "basal"a & 65 & 88 & 91 & 81 & 87 & 89 & 59 \\
\hline \multicolumn{8}{|l|}{ Amborella/Nymphaeaceae } \\
\hline basal $^{\mathrm{b}}$ & 72 & 98 & 98 & 98 & 98 & 95 & 99 \\
\hline \multicolumn{8}{|l|}{ Amborella/Nymphaeaceae/ } \\
\hline Austrobaileyales basal $^{c}$ & 71 & 96 & 98 & 98 & 98 & 93 & 99 \\
\hline Magnoliids & $<50$ & 62 & 78 & 86 & 77 & 65 & $<50$ \\
\hline Laurales + Magnoliales & $\ldots$ & 60 & 98 & 88 & 89 & 83 & 96 \\
\hline Canellales + Piperales & $\ldots$ & 80 & 75 & 88 & 90 & 75 & $<50$ \\
\hline Eudicots + Ceratophyllaceae & 53 & $\ldots$ & $\ldots$ & 74 & 73 & $\ldots$ & $\ldots$ \\
\hline Monocots + Ceratophyllaceae & $\ldots$ & $<50$ & 57 & $\ldots$ & $\ldots$ & 50 & 88 \\
\hline
\end{tabular}

Note. $<50$ indicates a clade that was retrieved with a data set but received bootstrap support $<50 \%$; ellipsis dots indicate a clade that was not retrieved with the data set indicated.

a Monophyly of all angiosperms other than Amborella.

b Monophyly of all angiosperms other than Amborella and Nymphaeaceae.

${ }^{c}$ Monophyly of all angiosperms other than Amborella, Nymphaeaceae, and Austrobaileyales.

angiosperms $(16 \mathrm{~A} \leftrightarrow \mathrm{G}$ and $22 \mathrm{C} \leftrightarrow \mathrm{T})$ and 33 sites showing transversions $(8 \mathrm{~A} \leftrightarrow \mathrm{C}, 8 \mathrm{~A} \leftrightarrow \mathrm{T}, 6 \mathrm{C} \leftrightarrow \mathrm{G}$, and $11 \mathrm{G} \leftrightarrow \mathrm{T}$ ). This substitution pattern and frequency clearly contrast with what would be expected if RNA editing and GC-content bias had contributed signal to link Amborella-NymphaeaceaeAustrobaileyales with the gymnosperms. RNA editing and reverse editing should result in far more changes of $\mathrm{C} \leftrightarrow \mathrm{T}$, $\mathrm{A} \leftrightarrow \mathrm{C}, \mathrm{A} \leftrightarrow \mathrm{T}, \mathrm{C} \leftrightarrow \mathrm{G}$, and $\mathrm{G} \leftrightarrow \mathrm{T}$ than $\mathrm{A} \leftrightarrow \mathrm{G}$ substitutions. The GC-content bias would predict many more changes of $\mathrm{A} \leftrightarrow \mathrm{G}, \mathrm{A} \leftrightarrow \mathrm{C}, \mathrm{G} \leftrightarrow \mathrm{T}$, and $\mathrm{C} \leftrightarrow \mathrm{T}$ than those of $\mathrm{A} \leftrightarrow \mathrm{T}$, and $\mathrm{C} \leftrightarrow \mathrm{G}$. For the five protein genes, only mitochondrial atp1 has all four sites located at the third codon positions, and the other four genes (plastid atpB, matK, $r b c L$, and mitochondrial $m a t R$ ) have sites at all three codon positions, with 11,8 , and 24 sites located at the first, second, and third codon positions, respectively. For the four rDNAs, all sites are located in well-aligned conservative regions. These results indicate that the phylogenetic signal in these nine genes that supports placement of Amborella, Nymphaeaceae, and Austrobaileyales as basal lineages is not likely due to any peculiar molecular evolutionary phenomena that may cause analytical artifacts, such as RNA editing and GC-content bias.

\section{Discussion}

Recent molecular analyses have converged on a topology of basal angiosperm relationships in which (1) Amborella, Nymphaeaceae, and Austrobaileyales represent the basal lineages of extant angiosperms; (2) two pairs of traditional magnoliid taxa, Magnoliales-Laurales and Canellales-Piperales, are sister to each other and form the magnoliid clade; and (3) Ceratophyllum, Chloranthaceae, monocots, magnoliids, and eudicots form a polytomy after the initial diversification that led to Amborella, Nymphaeaceae, and Austrobaileyales (Mathews and Donoghue 1999; Qiu et al. 1999, 2000; Graham and Olmstead 2000b; Soltis et al. 2000; Zanis et al. 2002, 2003; Borsch et al. 2003; Hilu et al. 2003; Löhne and Borsch 2005). This set of relationships has been used to formalize a classification system for angiosperms (APG II 2003 ) and to guide investigation of various aspects of early angiosperm evolution (e.g., Endress and Igersheim 2000; Friis et al. 2000; Thien et al. 2000; Williams and Friedman 2002; Ronse De Craene et al. 2003; Feild et al. 2004; Kramer et al. 2004). Work is still needed to establish firmly that the current consensus rests on a solid phylogenetic foundation and, more importantly, to resolve the polytomy among Ceratophyllum, Chloranthaceae, monocots, magnoliids, and eudicots. Attention to these pivotal issues in our understanding of the origin and early evolution of angiosperms is justified, especially given that three recent analyses using entire plastid genome sequences have failed to confirm that Amborella and Nymphaea are basal lineages in angiosperm phylogeny (Goremykin et al. 2003a, 2003b, 2004) and published molecular analyses have not obtained full resolution and strong support for most higher-level relationships among basal angiosperms. Below we discuss these issues.

Fig. 2 One of the two shortest trees found in the parsimony analysis of the five-protein-gene matrix. Numbers above branches are branch lengths (ACCTRAN optimization); those below in italics are bootstrap percentages (only those $>50 \%$ are shown; for branches related to Amborella, Nymphaeaceae, Austrobaileyales, Ceratophyllum, magnoliids, monocots, and eudicots, the bootstrap percentages are in boldface). The node labeled with an asterisk is collapsed in the strict consensus of the two shortest trees. Abbreviations: GYM=gymnosperms; $\mathrm{AMB}=$ Amborella $; \mathrm{NYM}=$ Nymphaeaceae $; \mathrm{AUS}=$ Austrobaileyales $; \mathrm{CHL}=$ Chloranthaceae $; \mathrm{CER}=$ Ceratophyllum $;$ MON $=$ monocots; $\mathrm{EUD}=$ eudicots; $\mathrm{CAN}=$ Canellales; $\mathrm{PIP}=$ Piperales; $\mathrm{MAG}=$ Magnoliales; $\mathrm{LAU}=$ Laurales; Acorus cal = Acorus calamus; Acorus gra $=$ Acorus gramineus; Ceratophyllum dem = Ceratophyllum demersum; Ceratophyllum sub = Ceratophyllum submersum. 

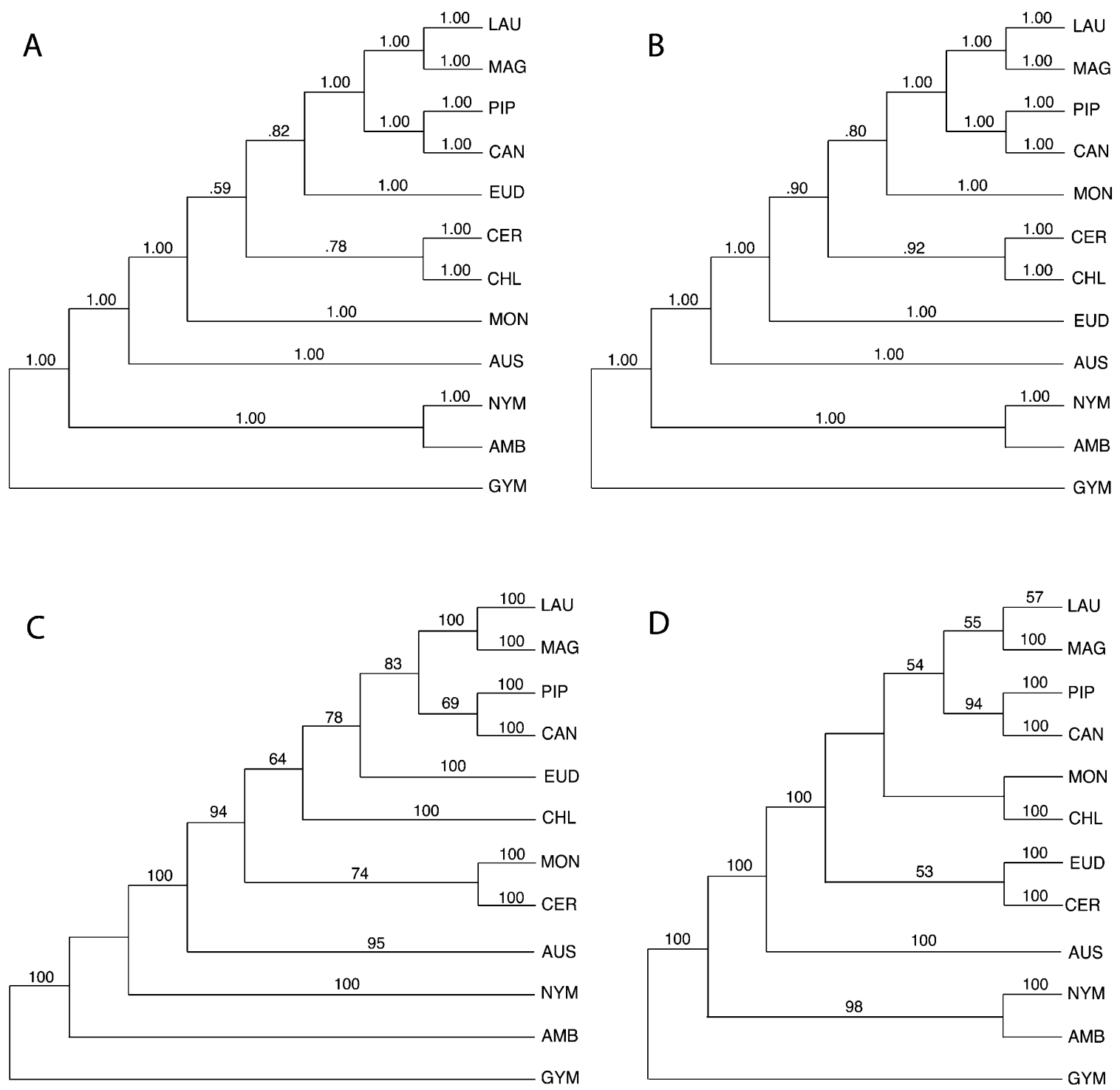

Fig. 3 Simplified presentation of the trees from Bayesian and fast maximum likelihood (ML) analyses of the nine-gene and five-protein-gene matrices. Taxa used in the analyses are the same as those used in figs. 1 and 2. A, Bayesian analysis of the nine-gene matrix. $B$, Bayesian analysis of the five-protein-gene matrix. C, ML analysis of the nine-gene matrix. $D$, ML analysis of the five-protein-gene matrix. The numbers above the branches are posterior probabilities for Bayesian analyses or bootstrap values for ML analyses. Abbreviations: GYM = gymnosperms; $\mathrm{AMB}=$ Amborella $; \mathrm{NYM}=$ Nymphaeaceae $; \mathrm{AUS}=$ Austrobaileyales $; \mathrm{CHL}=$ Chloranthaceae $; \mathrm{CER}=$ Ceratophyllum $;$ MON $=$ monocots; $\mathrm{EUD}=$ eudicots; $\mathrm{CAN}=$ Canellales; PIP $=$ Piperales; MAG $=$ Magnoliales; $\mathrm{LAU}=$ Laurales.

\section{Amborella, Nymphaeaceae, and Austrobaileyales as the Basalmost Lineages of Extant Angiosperms}

Several early studies hinted at the possibility that one or more of the three lineages now placed at the base of the angiosperm phylogenetic tree, Amborella, Nymphaeaceae, and Austrobaileyales, could represent the earliest-diverging lineages of extant angiosperms (Donoghue and Doyle 1989; Martin and Dowd 1991; Hamby and Zimmer 1992; Qiu et al. 1993; Soltis et al. 1997). However, lack of strong internal support and poor resolution in parts of the topologies prevented general acceptance of those results. In 1999-2000, several comprehensive analyses using extensive taxon and gene sampling as well as duplicate gene rooting strategy identified Amborella, Nymphaeaceae, and Austrobaileyales as the successive sister clades to all other angiosperms (Mathews and Donoghue 1999, 2000; Parkinson et al. 1999; Qiu et al. 1999, 2000; Soltis et al. 1999a; Barkman et al. 2000; Graham and Olmstead 2000b; Soltis et al. 2000). The impressively resolved overall topology with strong bootstrap support and a high degree of convergence of results from different research groups using different taxon and gene sampling schemes as well as different rooting strategies led to the realization that the earliest-diverging lineages of extant angiosperms had been identified. Subsequent analyses with different methods and 


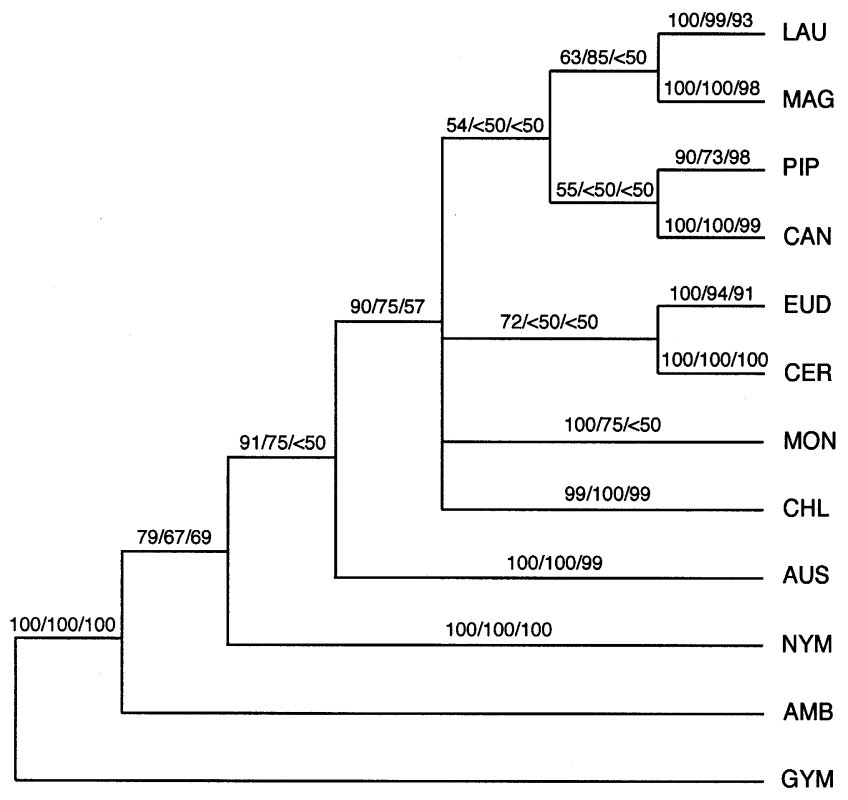

Fig. 4 Simplified presentation of parsimony bootstrap consensus trees of the three genome-specific analyses. Taxa used in the analyses are the same as those used in figs. 1 and 2. The three numbers above the branch separated by slashes are bootstrap values from plastid, mitochondrial, and nuclear genome-specific analyses, respectively. Abbreviations: $\mathrm{GYM}=$ gymnosperms; $\mathrm{AMB}=$ Amborella $; \mathrm{NYM}=$ Nymphaeaceae; $\quad$ AUS $=$ Austrobaileyales; $\mathrm{CHL}=$ Chloranthaceae; $\mathrm{CER}=$ Ceratophyllum $; \quad \mathrm{MON}=$ monocots; $\quad \mathrm{EUD}=$ eudicots; $\mathrm{CAN}=$ Canellales $; \quad \mathrm{PIP}=$ Piperales $; \quad \mathrm{MAG}=$ Magnoliales $; \quad \mathrm{LAU}=$ Laurales.

new data have further confirmed and reinforced this consensus (Qiu et al. 2001; Zanis et al. 2002, 2003; Borsch et al. 2003; Hilu et al. 2003; Löhne and Borsch 2005).

In contrast to this seemingly well-established earlier consensus, three recent analyses by Goremykin et al. (2003a, $2003 b$, 2004) using entire plastid genome sequences failed to place Amborella and Nymphaea as basal lineages of angiosperms. Although the scanty taxon sampling, particularly of monocots, which occupy the basalmost position among angiosperms in the trees obtained by these authors, raises doubt about the validity of their conclusions (Soltis and Soltis 2004; Soltis et al. 2004; Stefanovic et al. 2004), it is important that we scrutinize our own data and analyses to ensure that our conclusions are not biased by any analytical problem. Despite theoretical understanding of several longstanding issues in phylogenetics, such as long branch attraction (Felsenstein 1978) and the trade-off between taxon versus character sampling (Hillis 1996, 1998; Graybeal 1998; Soltis et al. 1998; Zwickl and Hillis 2002), it is still not clear how best to diagnose the effects of long branch attraction or inadequate taxon or character sampling in empirical studies. We have therefore conducted various kinds of analyses since our initial publications to detect any possible "misbehavior" of the data that might have contributed to the topology we obtained (cf. Qiu et al. 2000, 2001).

In this study, we further examined the substitutions separating gymnosperms-Amborella-Nymphaeaceae-Austrobaileyales from all other angiosperms and found that these changes are distributed in all nine genes from the three genomes and include all six possible substitutional changes at frequencies that do not seem to be biased by RNA editing or GC-content bias (fig. 5). This result, together with previously published tests (Qiu et al. 2000, 2001) that showed that the Amborella-Nymphaeaceae-Austrobaileyales rooting in our earlier analyses (Qiu et al. 1999) was unaffected by long branch attraction, suggests that the strategy of using multiple genes and dense "judicious" taxon sampling (Hillis 1998) is effective in tackling the recalcitrant problem of determining the earliest-diverging lineages of extant angiosperms.

In their most recent study, Goremykin et al. (2004) presented a comparison of putative synapomorphic substitutions between the Poaceae-basal or the Amborella-NymphaeaceaeAustrobaileyales-basal topologies and found that there are more sites supporting the former than the latter. We note that their use of a single gymnosperm (Pinus) as the outgroup, use of Poaceae as the only representatives of monocots, and exclusion of the third codon positions could lead to misidentification and underdetection of synapomorphic sites. In our analysis, we applied a more stringent criterion to score a site as synapomorphic; namely, it had to be conserved in at least two of the four gymnosperm lineages and two of $\mathrm{Am}$ borella, Nymphaeaceae, and Austrobaileyales but with a largely invariable different nucleotide in all other angiosperms. Furthermore, conservation of the five linked sites in the mtSSU, GTGTG in gymnosperms-Amborella-Nymphaeaceae (fig. 5) actually extends to Adiantum, Huperzia, and Lycopodium (Duff and Nickrent 1999) and possibly throughout all nonflowering land plants (Oda et al. 1992; Duff and Nickrent 1999; Parkinson et al. 1999; Chaw et al. 2000). Moreover, 28 of 47 sites that contain synapomorphic substitutions in the five protein genes are located at the third codon positions. Thus, we argue that the sites we identified are free of the problems of insufficient taxon sampling and bias and probably represent many of the sites that contain phylogenetic signal for resolving the basalmost angiosperm issue.

Finally, the placement of Amborella, Nymphaeaceae, and Austrobaileyales as basal lineages is supported by all three single-genome analyses (fig. 4), passing the test that a robust understanding of organismal phylogeny should be supported by analysis of all genomes within the organism (Qiu and Palmer 1999). Additionally, both the nine-gene and fiveprotein-gene analyses using parsimony, ML, and Bayesian methods give strong support to this topology. In consideration of the variety of analyses we have conducted on our multigene data set in this and previous studies (Qiu et al. 1999, 2000, 2001), it is safe to conclude that the AmborellaNymphaeaceae-Austrobaileyales-basal topology of the angiosperm phylogeny has been rigorously tested. Moreover, the congruent topologies inferred from functionally and structurally different coding genes in this study and others (e.g., phytochromes: Mathews and Donoghue 1999, 2000; floral MADS-box genes: Kim et al. 2004) and noncoding DNAs in the analyses of Borsch et al. (2003) and Löhne and Borsch (2005) should make sufficiently clear that locus-inherent specific patterns of molecular evolution have not led to a spurious conclusion of the rooting of angiosperm phylogeny. 




$223333233131321331133 \quad 3333231321213331311323 \quad 3333$

GTGGCACCGT CTACCGCCCTC AGTCAAAC tTtTATATTATATA AGGA CGGGA CAAAAC TCACtTA tAatcC

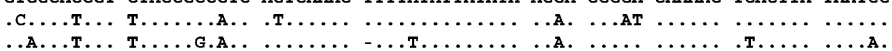

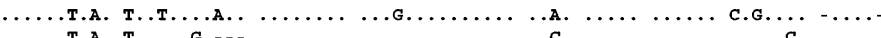

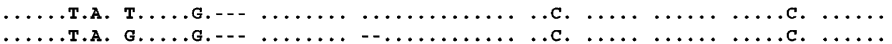

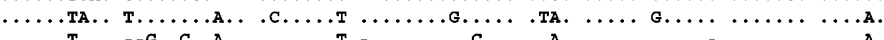

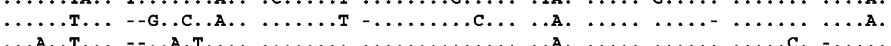

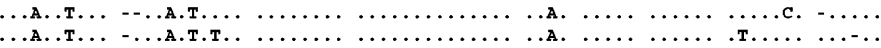

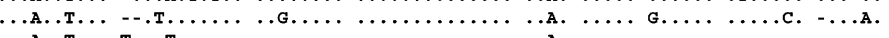

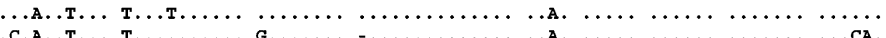

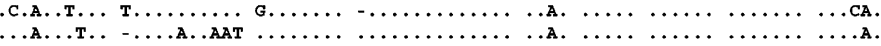


$\begin{array}{llllll}\ldots, \ldots & \ldots \ldots & \ldots\end{array}$ $\ldots$ AG.... T......

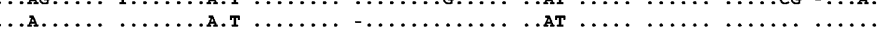

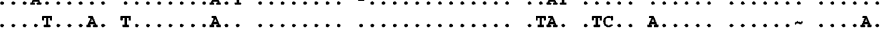



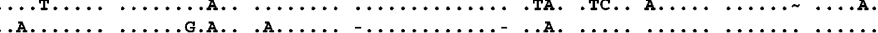

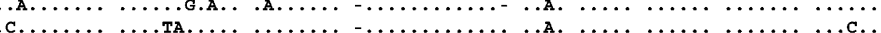

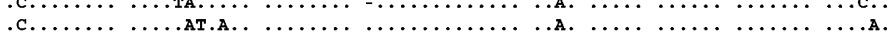



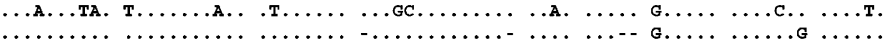

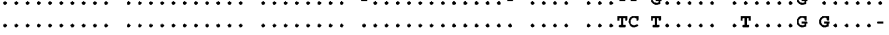



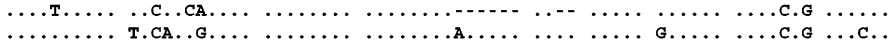



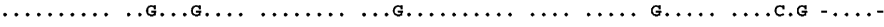

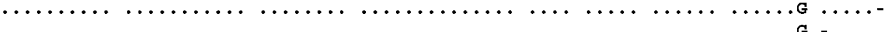

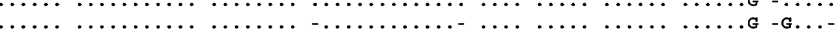

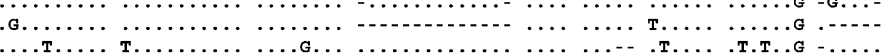

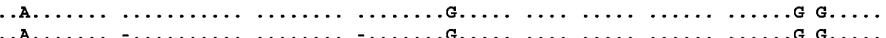

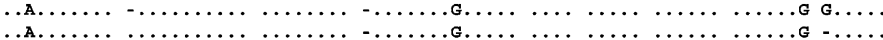

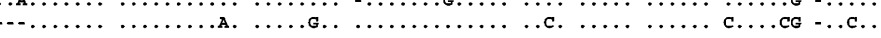

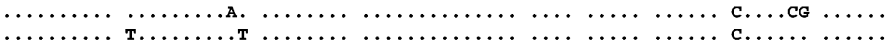

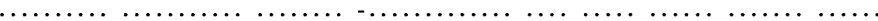

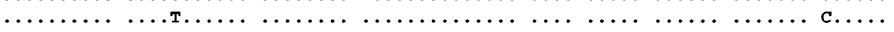

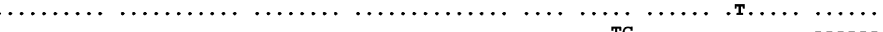
........ …..

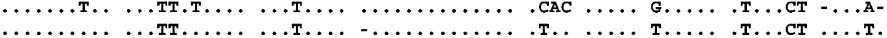

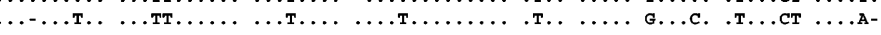

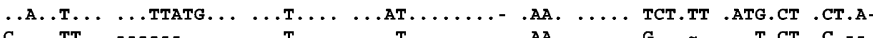
....тт..

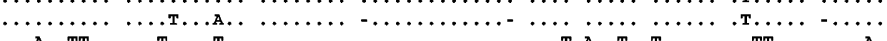
А..тт. $\ldots$ т. $\ldots$ т. $\ldots \ldots \ldots, \ldots \ldots \ldots \ldots \ldots \ldots$.

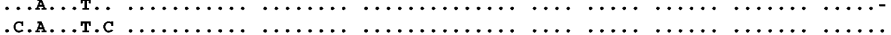

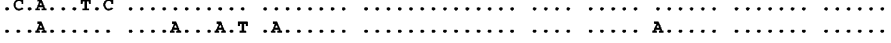

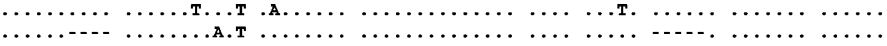

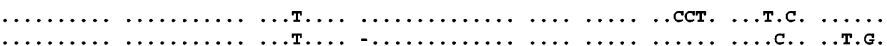
N.A...A. W.G.A.G. .AC.... .СА...

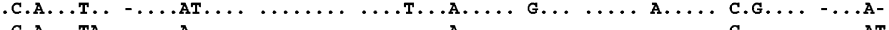

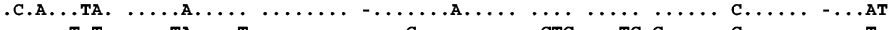

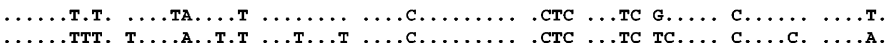

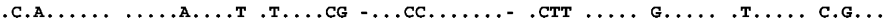

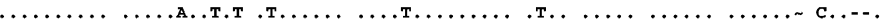





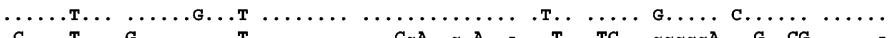
С.

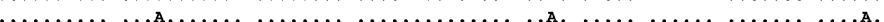

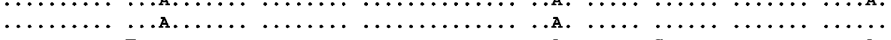


T.A.TG... T..TATGA.G GA.TGGA C..G.... . . G.T G.TT G.......... C.GT.CT GTTGG. T.A.TG.... TC.TATTGA.G GA.TGGG $\ldots$.......... T.A.TG.T. TC.TATGA.G GA.TGGA C..........


T..A.G.... TCGTAT.GA.G G...GGGA C..GC... ...G.T GTTT G.... .....G C.GT..T GTT.G. TC.AAGTIA. TCGTATIGA.A GACTGGGA CCCGCATGGCCGCT GTT GTCTC GTGTGG CTGT.CG GT..GT TC. AAGTTAC TCGTATTGA.A GACTGGGA CCCGCATGGCCGCT GTTT GTCTC GTGTGG CTGT.CG GT.AGTC.AAGTTAC TCGTATTGACA GACT.GGA CCCGCATGGCCGCT GTTW . TCTC GTGTGG CTGTCCG GC..G- . AAGTTAC TCGTAT--ACA GACT.GGA CCCACATGGCCGCT GTTT GTCTC GTGTGG CTGTCCG GC.AGT TCAAA.TTAC TCGTATTGACA GAC.GGGA -CCGCATGGCCGC- GT-- GTCTC GTGTGG CTGTCCG G.TGGT TAAAA.TTA. --.A.ATA.C. GACT.G.A CC.GCATGGC GC- GT.T GTC.G GTGTG. CTGTCCG G.TGGT TAAAA.TTAC ---ATATGTCA GA.T...A -C.GCATGGC GC- GATT GTC.G GTGTGG CTGTACG GTTGG, TCAAAGTTAC ---AATT.ACA GACTGGGA -CCGCATGGC GC- GTTT GTCTC GTGTGG C.GTCCC GTTGGT TCAAAGT-- T.GTA.TGACA GAGTG.G. - T.-.- C.G.CC- -TTGGT T AAAGT--- T.GTGT.GACA GACT.GGA --CGCATGGTCGC- G.TT GTCTC GTGTGG C.GTCCC GTTGGT TCAAGG.TAC --GTATTGACA GACTGGGA CCCACATGGCCGCT GTT- GTCTC GTGTGT CTGT..G -TTAGT TCAAGGTTAC TCGTATTGACA C.CTGGGA CCCACACGGCCGC- GTTW GTCTC GTGTGG CTGTCCG CTTAGT --AAGTTAC TCGTATTGACA GACTGGG CCCA.ACGGCCGCT GTTW GTCTC GTGTGG CTGTCCG GTTGGT atpB matk rbcL matR atp1 mtLsu mtSSU nu18S nu26s 


\section{Monophyly of and Relationships within the Magnoliids}

Initial support for the magnoliid clade (Qiu et al. 1999, 2000) was not strong, and morphological evidence was lacking (Doyle and Endress 2000). However, other analyses with different methods and data have consistently corroborated this finding (Mathews and Donoghue 1999; Barkman et al. 2000; Graham and Olmstead 2000b; Zanis et al. 2002, 2003; Borsch et al. 2003; Hilu et al. 2003). Recent analysis of the group II intron in petD also found a synapomorphic indel for the magnoliid clade (Löhne and Borsch 2005). The parsimony analysis of the five-protein-gene matrix in this study yielded strong bootstrap support for both monophyly of the magnoliids and relationships among the four member subclades (fig. 2). Further, Bayesian and ML analyses of both nine-gene and five-protein-gene matrices recovered this clade and resolved the same set of relationships, despite with varying PP and bootstrap values (fig. 3). Thus, it is reasonable to say that the magnoliids represent a major clade of basal angiosperms. The taxa included in this clade represent a majority of the traditional ranalian complex (Qiu et al. 1993). With Amborella, Nymphaeaceae, Austrobaileyales, Ceratophyllum, Chloranthaceae, Ranunculales, Papaverales, and Nelumbo removed, all other taxa of Cronquist's (1981) subclass Magnoliidae remain as magnoliids.

Identification of this large magnoliid clade significantly enhances clarification and will aid further resolution of relationships among basal angiosperms. It effectively reduces the options for placing Chloranthaceae, a family that has been placed previously with Laurales (Thorne 1992), Piperales (Cronquist 1981), and Canellales (Dahlgren 1989) and that is still uncertain for its phylogenetic affinity. Furthermore, placement of Piperales as sister to Canellales within the magnoliids removes the order from the list of taxa to be considered as potential sister lineages to monocots, as Burger (1977) suggested a close relationship between Piperales and monocots. Similarly, Magnoliales (termed as Annonales then) alone can no longer be entertained as a potential sister group to monocots, as proposed by Dahlgren et al. (1985), since they are embedded within the magnoliid clade.

The close relationship between Magnoliales and Laurales was clearly recognized in the premolecular systematics era (Cronquist 1981). Two genome-specific analyses (plastid and mitochondrial), the nine-gene analysis, and the five-proteingene analysis all identified this relationship, generally with strong support (figs. 1-4). Winteraceae and Canellaceae (collectively classified as Canellales; APG II 2003), traditionally placed in Magnoliales (Cronquist 1981) and still associated with that order in a morphological cladistic analysis by Doyle and Endress (2000), consistently appear as the sister to Piperales. The two larger clades, Magnoliales-Laurales and
Canellales-Piperales, are sister to each other in all analyses that recovered the magnoliid clade (Mathews and Donoghue 1999; Graham and Olmstead 2000b; Zanis et al. 2002, 2003; Borsch et al. 2003; Hilu et al. 2003). Hence, these relationships among the magnoliid lineages can be deemed robust. However, they are different from those depicted by a morphological cladistic analysis (Doyle and Endress 2000). Convergence at the morphological level may be a factor. Future investigations of the development of morphological characters using molecular genetic approaches (e.g., Buzgo et al. 2004; Kramer et al. 2004) and other nonmolecular characters may sort out homoplasy and identify proper synapomorphies for the several clades identified here.

\section{Relationships among Ceratophyllum, Chloranthaceae, Monocots, Magnoliids, and Eudicots}

The primary remaining challenge is to resolve relationships among Ceratophyllum, Chloranthaceae, monocots, magnoliids, and eudicots. The highly divergent nature of Ceratophyllum was noticed by Les and his colleagues as early as 1988 and 1991, based on both morphological and molecular evidence. The phylogenetic affinity of this genus remains elusive. Based on bootstrap support for the placement of Ceratophyllum, which is moderate at best, our nine-gene and five-protein-gene analyses present two alternative hypotheses on the placement of the genus, sister to monocots and eudicots, respectively (figs. 1, 2; fig. 3C, 3D). The relationship of Ceratophyllum to eudicots was reported by Soltis et al. (2000) with only 53\% jackknife support, by Hilu et al. (2003) with $71 \%$ jackknife support, and by Graham et al. (forthcoming) with $82 \%$ bootstrap support. The $74 \%$ parsimony bootstrap value and $53 \%$ ML bootstrap value in our five-protein-gene analyses (fig. 2) support this relationship to eudicots. In contrast, the placement with the monocots supported by our nine-gene analysis using both parsimony and ML methods is undermined by a topological anomaly within the monocots, i.e., the sister relationship of Acorus to alismatids (fig. 1). The correct placement of Acorus is sister to all other monocots according to several analyses with a large monocot sampling (Chase et al. 2000, forthcoming; Soltis et al. 2000; Hilu et al. 2003). The erroneous position of Acorus here could indicate that the placement of Ceratophyllum in the nine-gene analysis is an artifact. Indeed, for all four mitochondrial genes we used (atp1, matR, mtSSU, and mtLSU), Ceratophyllum, Acorus, and alismatids have highly divergent sequences in comparison to other basal angiosperms, indicating that they could attract to each other as long branches. The relationship of Ceratophyllum to Chloranthaceae, shown by our Bayesian analyses of both the nine-gene

Fig. 5 "Synapomorphic substitutions" that separate gymnosperms-Amborella-Nymphaeaceae-Austrobaileyales (or just Amborella and Nymphaeaceae in some cases) from all other angiosperms in plastid atpB, mat $K$, and $r b c L$, mitochondrial matR, atp1, mtLSU, and mtSSU, and nuclear $18 \mathrm{~S}$ and $26 \mathrm{~S}$ rDNAs. The numbers in the top row refer to codon positions in the protein genes. A hyphen indicates missing data; a tilde $(\sim)$ indicates a gap; dots denote the same nucleotides as in Magnolia (the top sequence). The underlined sites are contiguous in the original alignment, and all other sites are distributed individually throughout the gene. Abbreviations: $\mathrm{GYM}=$ gymnosperms; $\mathrm{AMB}=$ Amborella; $\mathrm{NYM}=$ Nymphaeaceae; $\quad$ AUS $=$ Austrobaileyales $; \quad \mathrm{CHL}=$ Chloranthaceae $; \quad \mathrm{CER}=$ Ceratophyllum $; \quad \mathrm{MON}=$ monocots; $\mathrm{EUD}=$ eudicots; $\mathrm{CAN}=$ Canellales; PIP $=$ Piperales; MAG $=$ Magnoliales; LAU $=$ Laurales. 
$(\mathrm{PP}=0.78)$ and five-protein-gene $(\mathrm{PP}=0.92)$ matrices (fig. 3 ), has been reported only once before (Antonov et al. 2000) and is difficult to evaluate, particularly given the current controversy surrounding the confidence one can have in the PP in Bayesian phylogenetics (Suzuki et al. 2002; Douady et al. 2003; Felsenstein 2004; Simmons et al. 2004).

The placement of Chloranthaceae among other basal angiosperms has long been a subject of debate (Qiu et al. 1993). Our nine-gene and five-protein-gene analyses did not yield bootstrap support to place this family with confidence (figs. 1, 2). Clearly, more work is needed to determine the phylogenetic affinity of this family.

Relationships among magnoliids, monocots, and eudicots, the three lineages encompassing nearly $3 \%, 22 \%$, and $75 \%$ of all angiosperm species diversity, respectively (Drinnan et al. 1994), continue to elude resolution despite several large-scale sequence analyses (Soltis et al. 2000; Savolainen et al. 2000; Hilu et al. 2003). Monocots were placed in a clade with magnoliids and Chloranthaceae with $56 \%$ jackknife support in Soltis et al. (2000), and this topology was also recovered by Hilu et al. (2003) using a different data set in a Bayesian analysis (but not in their parsimony analysis). Eudicots-Ceratophyllum were sister to this large clade. Our Bayesian analysis of the five-protein-gene matrix obtained a similar topology, with Ceratophyllum placed as a sister to Chloranthaceae instead of eudicots (fig. 3). Alternatively, the eudicots-Ceratophyllum clade is sister to the magnoliids in our five-protein-gene parsimony analysis, but without bootstrap support $>50 \%$ (fig. 2). A similar topology was obtained in our earlier studies using a slightly different data set (Qiu et al. 1999, 2000) with the exception that Ceratophyllum was not placed with eudicots but rather with monocots. The third possible arrangement for these three large angiosperm lineages, with monocots and eudicots as sister to each other, has been seen in three analyses of plastid and nuclear genes (Mathews and Donoghue 1999; Graham and Olmstead 2000b; Graham et al., forthcoming). Thus, all three possible arrangements for monocots, magnoliids, and eudicots have been observed. It is clear that more data, in terms of both character and taxon sampling (particularly of monocots and eudicots), are needed before a firm conclusion can be reached on relationships among these three large angiosperm lineages.

\section{Conclusions}

Our analyses, as well as several earlier studies of the angiosperm phylogeny, revealed a steady increase in resolution and internal support for relationships as genes were added to initial single-gene matrices to form multigene data sets. For example, Soltis et al. (1998) revealed a steady increase in support for angiosperm relationships (including basal angiosperm relationships) as sequences from $18 \mathrm{~S} \mathrm{rDNA}$ and atpB were added to an $r b c L$ data matrix to form two and threegene data sets (also Soltis et al. 1999a, 2000; Savolainen et al. 2000; table 3). Similarly, Qiu et al. (1999, 2000) also observed an increase in the support for basal angiosperm relationships in an analysis of a five-gene data set (table 3). Support for many critical relationships among basal angiosperms continued to increase in the analyses of Zanis et al. (2002), which involved a matrix of five to 11 genes. In this study, phylogenetic analysis of the five protein-coding genes (atpB, matK, rbcL, atp1, and matR) yielded a topology and internal support for relationships generally comparable to those realized in the earlier multigene analysis of Zanis et al. (2002), with the exception of Ceratophyllum, which was placed differently in the two studies. Much of the increase in internal support from these five protein-coding genes compared to the five-gene analysis of Qiu et al. (1999), based on $a t p B, 18 \mathrm{~S}, r b c L$, atp1, and matR, involves the signal provided by matK. In fact, the rapidly evolving matK alone provides resolution and support comparable to that achieved with three more slowly evolving genes, $r b c L, 18 \mathrm{~S}$, and atpB (Hilu et al. 2003). Our analyses indicate that the addition of the two nuclear rDNAs does not increase the support for most of the critical nodes we examined (table 3). For example, the addition of $18 \mathrm{~S}$ did increase the support for the placement of Amborella as sister to all other flowering plants, but conversely, the support for the magnoliid clade was somewhat lower than that achieved with the five protein-coding genes. The addition of $26 \mathrm{~S}$ slightly increased support for the placement of Amborella, but support for the monophyly of the magnoliid clade and Canellales + Piperales both decreased compared to the five-protein-gene analysis. The placement of Ceratophyllum also changed with the addition of $26 \mathrm{~S}$ (table 3 ).

The most dramatic change in the internal support for clades resulted from the addition of the two mitochondrial rDNAs. The addition of these two genes resulted in a sharp drop in the support for Amborella as sister to all other angiosperms (to $59 \%$ ), with support for the monophyly of magnoliids and also of Canellales + Piperales dropping below 50\%. These two mitochondrial genes appear to be adding conflicting signal to that from the protein-coding and nuclear $18 \mathrm{~S}$ rDNA. Conflict is also evident among data sets regarding the placement of Ceratophyllum as sister to either eudicots or monocots (table 3 ). The conflict introduced by $\mathrm{mtSSU}$ and mtLSU with regard to monophyly of magnoliids and relationships among their member clades seems to be caused by lineage-specific rate heterogeneity in these two genes (data not shown), whereas the drop in support for Amborella as the sister to all other angiosperms after addition of these two genes reflects a genuine uncertainty on the exact topology at the first node in the angiosperm phylogeny, as Amborella and Nymphaeaceae together are supported as the earliestdiverging lineage in three of the six analyses performed in this study (fig. 3; Barkman et al. 2000; Qiu et al. 2000; Stefanovic et al. 2004). More data are clearly needed to resolve this kind of conflict among different genes.

The comparisons we have conducted (table 3) provide a valuable lesson in the addition of genes. Although total evidence is a preferred approach (Soltis et al. 1998, 2000; Qiu et al. 1999; Savolainen et al. 2000), with some investigators advocating the combination of many genes (Rokas et al. 2003), it is important to stress that not all genes contain the same amount of information for phylogenetic reconstruction (Hilu et al. 2003) and that not all genes have the same history (Maddison 1997). These gene-specific effects are caused by differences in size and internal mutational dynamics and have to be considered in addition to well-known effects of different evolutionary histories caused by reticulations or 
lineage sorting. Although total evidence is encouraged, it is important to evaluate the contribution and impact of individual genes. In our analyses, for example, the addition of two mitochondrial and nuclear 26S rDNAs had a negative impact on resolution and support for certain parts of the tree. Chase et al. (forthcoming) also observed that in a seven-gene combined analysis of monocots, the addition of $18 \mathrm{~S}$ and partial $26 \mathrm{~S}$ did not increase support and for some clades resulted in weaker support than a combined analysis of protein-coding plastid genes. Therefore, the total evidence approach needs to be taken with caution.

Besides amassing multigene sequence data for a large number of taxa, a different approach also promises to resolve the relationships among major angiosperm lineages, i.e., to search for informative genomic structural changes such as those reported for resolving the origin of and relationships within land plants (Manhart and Palmer 1990; Raubeson and Jansen 1992; Qiu et al. 1998; Lee and Manhart 2002; Dombrovska and Qiu 2004; Qiu and Palmer 2004; Quandt et al. 2004; Löhne and Borsch 2005). This approach is especially promising given that the entire plastid genome from an increasing number of angiosperms and other land plants has been sequenced (Goremykin et al. 2003a, 2003b; 2004), and more work is in progress. However, caution must be taken to ensure an appropriate taxonomic coverage so that homologous changes can be distinguished from homoplasious ones (Qiu and Palmer 2004).

Therefore, we recommend that future efforts be directed toward exploration of more data, for both sequences and gene/genome structural features, with proper attention paid to both quality and quantity of taxon and character sam- pling. The most effective and efficient ways to analyze the resulting large matrices remain parsimony methods, which have been shown to be robust even when data are heterogeneous (Kolaczkowski and Thornton 2004). Bayesian bootstrapping (Douady et al. 2003), when it can be practically implemented, will also be worth pursuing on these large matrices. The fast ML method developed by Guindon and Gascuel (2003) provides a third possibility for analyzing large data matrices as demonstrated in this study. Careful evaluation of support values using bootstrapping or jackknifing (internal support, Nei et al. 1998) as well as congruence with other evidence (external support, Chase et al. 1993; taxonomic congruence, Miyamoto and Fitch 1995) will be essential to ensure correct interpretation of analytical results.

\section{Acknowledgments}

We thank James A. Doyle for stimulating discussion and Tory Hendry for technical assistance. The computation was partially conducted using the GCRC computation resources of the University of Michigan funded by National Institutes of Health (NIH) grant M01 RR00042. D. E. Soltis and P. S. Soltis were supported by National Science Foundation (NSF) Assembling the Tree of Life (AToL) grant DEB 0431266. R. R. Gutell and J. J. Cannone were supported by a grant from the NIH (GM 067317). C. C. Davis was supported by NSF AToL grant EF 04-31242 and by the Michigan Society of Fellows. Y.-L. Qiu was supported by an Early Career award (DEB 0332298) and an AToL grant (DEB 0431239) from the NSF and a research grant from the Swiss National Fund (3100-053602).

\section{Literature Cited}

Antonov AS, AV Troitsky, TKH Samigullin, VK Bobrova, KM Valiejo-Roman, W Martin 2000 Early events in the evolution of angiosperms deduced from cp rDNA ITS2 sequence comparisons. Pages 210-214 in Proceedings of the International Symposium on the Family Magnoliaceae, May 18-22, 1998, Guangzhou, China. Science, Beijing.

APG II (Angiosperm Phylogeny Group II) 2003 An update of the Angiosperm Phylogeny Group classification for the orders and families of flowering plants: APG II. Bot J Linn Soc 141: 399-436.

Azuma H, LB Thien, S Kawano 1999 Molecular phylogeny of Magnolia (Magnoliaceae) inferred from cpDNA sequence and evolutionary divergence of the floral scents. J Plant Res 112: 291-306.

Barkman TJ, G Chenery, JR McNeal, J Lyons-Weiler, WJ Ellisens, G Moore, AD Wolfe, CW dePamphilis 2000 Independent and combined analyses of sequences from all three genomic compartments converge on the root of flowering plant phylogeny. Proc Natl Acad Sci USA 97:13166-13171.

Bergthorsson U, KL Adams, B Thomason, JD Palmer 2003 Widespread horizontal transfer of mitochondrial genes in flowering plants. Nature 424:197-201.

Borsch T, KW Hilu, D Quandt, V Wilde, C Neinhuis, W Barthlott 2003 Noncoding plastid trnT-trnF sequences reveal a well resolved phylogeny of basal angiosperms. J Evol Biol 16: 558-576.

Bowe LM, G Coat, CW dePamphilis 2000 Phylogeny of seed plants based on all three plant genomic compartments: extant gymno- sperms are monophyletic and Gnetales are derived conifers. Proc Natl Acad Sci USA 97:4092-4097.

Burger WC 1977 The Piperales and the monocots: alternative hypotheses for the origin of monocotyledonous flowers. Bot Rev 43:345-393.

Buzgo M, DE Soltis, PS Soltis, H Ma 2004 Towards a comprehensive integration of morphological and genetic studies of floral development. Trends Plant Sci 7:22-31.

Caddick LR, PJ Rudall, P Wilkin, TAJ Hedderson, MW Chase 2002 Phylogenetics of Dioscoreales based on combined analyses of morphological and molecular data. Bot J Linn Soc 138: 123-144.

Chase MW, MF Fay, DS Devey, O Maurin, N Rønsted, J Davies, Y Pillon, et al Forthcoming Multi-gene analyses of monocot relationships: a summary. In JT Columbus, EA Friar, CW Hamilton, JM Porter, LM Prince, MG Simpson, eds. Monocots: comparative biology and evolution. 2 vols. Rancho Santa Ana Botanic Garden, Claremont, CA.

Chase MW, DE Soltis, RG Olmstead, D Morgan, DH Les, BD Mishler, MR Duvall, et al 1993 Phylogenetics of seed plants: an analysis of nucleotide sequences from the plastid gene $r b c \mathrm{~L}$. Ann Mo Bot Gard 80:528-580.

Chase MW, DE Soltis, PS Soltis PJ Rudall, MF Fay, WH Hahn, S Sullivan, et al 2000 Higher-level systematics of the monocotyledons: an assessment of current knowledge and a new classification. Pages 3-16 in KL Wilson, DA Morrison, eds. Monocots: systematics and evolution. CSIRO, Melbourne.

Chaw S-M, CL Parkinson, Y Cheng, TM Vincent, JD Palmer 2000 
Seed plant phylogeny inferred from all three plant genomes: monophyly of extant gymnosperms and origin of Gnetales from conifers. Proc Natl Acad Sci USA 97:4086-4091.

Chaw S-M, A Zharkikh, H-M Sung, T-C Lau, W-H Lee 1997 Molecular phylogeny of extant gymnosperms and seed plant evolution: analysis of nuclear 18S rRNA sequences. 14:56-68.

Crane PR 1989 Paleobotanical evidence on the early radiation of nonmagnoliid dicotyledons. Plant Syst Evol 162:165-191.

Cronquist A 1981 An integrated system of classification of flowering plants. Columbia University Press, New York.

Dahlgren G 1989 The last Dahlgrenogram system of classification of the dicotyledons. Pages 249-260 in K Tan, ed. Plant taxonomy, phytogeography and related subjects. Edinburgh University Press, Edinburgh.

Dahlgren R, K Bremer 1985 Major clades of the angiosperms. Cladistics 1:349-368.

Dahlgren R, HT Clifford, PF Yeo 1985 The families of the monocotyledons: structure, evolution, and taxonomy. Springer, Berlin.

Dombrovska O, Y-L Qiu 2004 Distribution of introns in the mitochondrial gene nad1 in land plants: phylogenetic and molecular evolutionary implications. Mol Phylogenet Evol 32:246-263.

Donoghue MJ, JA Doyle 1989 Phylogenetic analysis of angiosperms and the relationships of Hamamelidae. Pages 17-45 in PR Crane, S Blackmore, eds. Evolution, systematics, and fossil history of the Hamamelidae. Vol 1. Clarendon, Oxford.

Douady CJ, F Delsuc, Y Boucher, WF Doolittle, EJP Douzery 2003 Comparison of Bayesian and maximum likelihood bootstrap measures of phylogenetic reliability. Mol Biol Evol 20:248-254.

Doyle JA, PK Endress 2000 Morphological phylogenetic analysis of basal angiosperms: comparison and combination with molecular data. Int J Plant Sci 161(suppl):S121-S153.

Doyle JA, CL Hotton 1991 Diversification of early angiosperm pollen in a cladistic context. Pages 169-195 in S Blackmore, SH Barnes, eds. Pollen and spores. Clarendon, Oxford.

Drinnan AN, PR Crane, SB Hoot 1994 Patterns of floral evolution in the early diversification of non-magnoliid dicotyledons (eudicots). Plant Syst Evol Suppl 8:93-122.

Duff RJ, DL Nickrent 1999 Phylogenetic relationships of land plants using mitochondrial small-subunit rDNA sequences. Am J Bot 86: 372-386.

Endress PK, A Igersheim 2000 Gynoecium structure and evolution in basal angiosperms. Int J Plant Sci 161(suppl):S211-S223.

Feild TS, NC Arens, JA Doyle, TE Dawson, MJ Donoghue 2004 Dark and disturbed: a new image of early angiosperm ecology. Paleobiology 30:82-107.

Felsenstein J 1978 Cases in which parsimony and compatibility methods will be positively misleading. Syst Zool 27:401-410. 2004 Inferring phylogenies. Sinauer, Sunderland, MA.

Fishbein M, C Hibsch-Jetter, DE Soltis, L Hufford 2001 Phylogeny of Saxifragales (angiosperms, tricolpates): analysis of a rapid, ancient radiation. Syst Biol 50:817-847.

Friis EM, KR Pedersen, PR Crane 2000 Reproductive structure and organization of basal angiosperms from the Early Cretaceous (Barremian or Aptian) of western Portugal. Int J Plant Sci 161(suppl):S169-S182.

Fuse S, MN Tamura 2000 A phylogenetic analysis of the plastid matK gene with emphasis on Melanthiaceae sensu lato. Plant Biol 2: 415-427.

Gadek PA, DL Alpers, MM Heslewood, CJ Quinn 2000 Relationships within Cupressaceae sensu lato: a combined morphological and molecular approach. Am J Bot 87:1044-1057.

Goremykin VV, KI Hirsch-Ernst, S Wölfl, FH Hellwig 2003a Analysis of the Amborella trichopoda plastid genome sequence suggests that Amborella is not a basal angiosperm. Mol Biol Evol 20: 1499-1505.
$2003 \mathrm{~b}$ The plastid genome of the "basal" angiosperm Calycanthus fertilis: structural and phylogenetic analyses. Plant Syst Evol 242:119-135.

2004 The plastid genome of Nymphaea alba, whole genome analyses and the problem of identifying the most basal angiosperm. Mol Biol Evol 21:1445-1454.

Graham SW, RG Olmstead 2000a Evolutionary significance of an unusual plastid DNA inversion found in two basal angiosperm lineages. Curr Genet 37:183-188.

$2000 b$ Utility of 17 plastid genes for inferring the phylogeny of the basal angiosperms. Am J Bot 87:1712-1730.

Graham SW, JM Zgurski, MA McPherson, DM Cherniawsky, JM Saarela, ESC Horne, SY Smith, et al Forthcoming Robust inference of monocot deep phylogeny using an expanded multigene plastid data set. In JT Columbus, EA Friar, CW Hamilton, JM Porter, LM Prince, MG Simpson, eds. Monocots: comparative biology and evolution. 2 vols. Rancho Santa Ana Botanic Garden, Claremont, CA.

Graybeal A 1998 Is it better to add taxa or characters to a difficult phylogenetic problem? Syst Biol 47:9-17.

Guindon S, O Gascuel 2003 A simple, fast and accurate method to estimate large phylogenies by maximum-likelihood. Syst Biol 52: 696-704.

Hamby RK, EA Zimmer 1992 Ribosomal RNA as a phylogenetic tool in plant systematics. Pages 50-91 in PS Soltis, DE Soltis, JJ Doyle, eds. Molecular systematics of plants. Chapman \& Hall, New York.

Hillis DM 1996 Inferring complex phylogenies. Nature 383:130.

1998 Taxon sampling, phylogenetic accuracy, and investigator bias. Syst Biol 47:3-8.

Hillis DM, JJ Bull 1993 An empirical test of bootstrapping as a method for assessing confidence in phylogenetic analysis. Syst Biol 42:182-192.

Hilu KW, T Borsch, K Müller, DE Soltis, PS Soltis, V Savolainen, MW Chase, et al 2003 Angiosperm phylogeny based on matK sequence information Am J Bot 90:1758-1776.

Hoot SB, A Douglas 1998 Phylogeny of the Proteaceae based on $a t p B$ and $a t p B / r b c L$ intergenic spacer region sequences Aust $\mathrm{J}$ Bot 11:301-320.

Hoot SB, S Magallon, PR Crane 1999 Phylogeny of basal tricolpates based on three molecular data sets: $a t p B, r b c L$, and $18 \mathrm{~S}$ nuclear ribosomal DNA sequences. Ann Mo Bot Gard 86:1-32.

Huelsenbeck JP, F Ronquist 2001 MrBayes: Bayesian inference of phylogeny. Bioinformatics 17:754-755.

Judd WS, RG Olmstead 2004 A survey of tricolpate (eudicot) phylogenetic relationships. Am J Bot 91:1627-1644.

Kim S, M-J Yoo, VA Albert, JS Farris, PS Soltis, DE Soltis 2004 Phylogeny and diversification of B-function MADS-box genes in angiosperms: evolutionary and functional implications of a 260million-year-old duplication. Am J Bot 91:2102-2118.

Kolaczkowski B, JW Thornton 2004 Performance of maximum parsimony and likelihood phylogenetics when evolution is heterogeneous. Nature 431:980-984.

Kramer EM, MA Jaramillo, VS Di Stilio 2004 Patterns of gene duplication and functional evolution during the diversification of the AGAMOUS subfamily of MADS box genes in angiosperms. Genetics 166:1011-1023.

Kugita M, Y Yamamoto, T Fujikawa, T Matsumoto, K Yoshinaga 2003 RNA editing in hornwort plastids makes more than half the genes functional. Nucleic Acids Res 31:2417-2423.

Kuzoff RK, JA Sweere, DE Soltis, PS Soltis, EA Zimmer 1998 The phylogenetic potential of entire $26 \mathrm{~S}$ rDNA sequence in plants. Mol Biol Evol 15:251-263.

Larsen N, GJ Olsen, BL Maidak, MJ McCaughey, R Overbeek, TJ Macke, TL Marsh, CR Woese 1993 The ribosomal database project. Nucleic Acids Res 21:3021-3023. 
Lee J, JR Manhart 2002 Four embryophyte introns and $p s b B$ operon indicate Chlorokybus as a basal streptophyte lineage. Algae 17: 53-58.

Les DH 1988 The origin and affinities of the Ceratophyllaceae. Taxon 37:326-435.

Les DH, MA Cleland, M Waycott 1997 Phylogenetic studies in Alismatidae. II. Evolution of marine angiosperms (seagrasses) and hydrophily. Syst Bot 22:443-463.

Les DH, DK Garvin, CF Wimpee 1991 Molecular evolutionary history of ancient aquatic angiosperms. Proc Natl Acad Sci USA 88:10119-10123.

Les DH, EL Schneider, DJ Pagett, PS Soltis, DE Soltis, M Zanis 1999 Phylogeny, classification and floral evolution of water lilies (Nymphaeaceae; Nymphaeales): a synthesis of non-molecular, $r b c L$, matK, and 18S rDNA data. Syst Bot 24:28-46.

Loconte H, DW Stevenson 1991 Cladistics of the Magnoliidae. Cladistics 7:267-296.

Löhne C, T Borsch 2005 Molecular evolution and phylogenetic utility of the petD group II intron: a case study in basal angiosperms. Mol Biol Evol 22:317-332.

Maddison WP 1997 Gene trees in species trees. Syst Biol 46: 523-536.

Maddison WP, DR Maddison 2002 MacClade: analysis of phylogeny and character evolution. Version 4.05. Sinauer, Sunderland, MA.

Manhart JR, JD Palmer 1990 The gain of two plastid tRNA introns marks the green algal ancestors of land plants. Nature 345: 268-270.

Martin PG, JM Dowd 1991 Studies of angiosperm phylogeny using protein sequences. Ann Mo Bot Gard 78:296-337.

Mathews S, MJ Donoghue 1999 The root of angiosperm phylogeny inferred from duplicate phytochrome genes. Science 286:947-950. 2000 Basal angiosperm phylogeny inferred from duplicated phytochromes A and C. Int J Plant Sci 161(suppl):S41-S55.

Minin V, Z Abdo, P Joyce, J Sullivan 2003 Performance-based selection of likelihood models for phylogeny estimation. Syst Biol 52:674-683.

Miyamoto MM, WM Fitch 1995 Testing species phylogenies and phylogenetic methods with congruence. Syst Biol 44:64-76.

Murata J, T Ohi, SG Wu, D Darnaedi, T Sugawara, T Nakanishi, H Murata 2001 Molecular phylogeny of Aristolochia (Aristolochiaceae) inferred from matK sequences. Acta Phytotaxon Geobot 52: 75-83.

Nairn CJ, RJ Ferl 1988 The complete nucleotide sequence of the small-subunit ribosomal RNA coding region for the cycad Zamia pumila: phylogenetic implications. J Mol Evol 27:133-141.

Nandi OI, MW Chase, PK Endress 1998 A combined cladistic analysis of angiosperms using $r b c L$ and nonmolecular data sets. Ann Mo Bot Gard 85:137-212.

Nei M, S Kumar, K Takahashi 1998 The optimization principle in phylogenetic analysis tends to give incorrect topologies when the number of nucleotides or amino acids used is small. Proc Natl Acad Sci USA 95:12390-12397.

Nickrent DL, A Blarer, Y-L Qiu, DE Soltis, PS Soltis, M Zanis 2002 Molecular data place Hydnoraceae with Aristolochiaceae. Am J Bot 89:1809-1817.

Oda K, K Yamato, E Ohta, Y Nakamura, M Takemura, N Nozato, K Akashi, et al 1992 Gene organization deduced from the complete sequence of liverwort Marchantia polymorpha mitochondrial DNA: a primitive form of plant mitochondrial genome. J Mol Biol 223:1-7.

Parkinson CL, KL Adams, JD Palmer 1999 Multigene analyses identify the three earliest lineages of extant flowering plants. Curr Biol 9:1485-1488.

Posada D, KA Crandall 1998 MODELTEST: testing the model of DNA substitution. Bioinformatics 14:817-818.

Pryer KM, H Schneider, AR Smith, R Cranfill, PG Wolf, JS Hunt, SD
Sipes 2001 Horsetails and ferns are a monophyletic group and the closest living relatives to seed plants. Nature 409:618-622.

Qiu Y-L, MW Chase, DH Les, CR Parks 1993 Molecular phylogenetics of the Magnoliidae: cladistic analyses of nucleotide sequences of the plastid gene $r b c \mathrm{~L}$. Ann Mo Bot Gard 80:587-606.

Qiu Y-L, Y Cho, JC Cox, JD Palmer 1998 The gain of three mitochondrial introns identifies liverworts as the earliest land plants. Nature 394:671-674.

Qiu Y-L, J Lee, F Bernasconi-Quadroni, DE Soltis, PS Soltis, M Zanis, EA Zimmer, Z Chen, V Savolainen, MW Chase 1999 The earliest angiosperms: evidence from mitochondrial, plastid and nuclear genomes. Nature 402:404-407.

2000 Phylogeny of basal angiosperms: analyses of five genes from three genomes. Int J Plant Sci 161(suppl):S3-S27.

Qiu Y-L, J Lee, BA Whitlock, F Bernasconi-Quadroni, O Dombrovska 2001 Was the ANITA rooting of the angiosperm phylogeny affected by long branch attraction? Mol Biol Evol 18:1745-1753.

Qiu Y-L, JD Palmer 1999 Phylogeny of basal land plants: insights from genes and genomes. Trends Plant Sci 4:26-30.

2004 Many independent origins of trans splicing of a plant mitochondrial group II intron. J Mol Evol 59:80-89.

Quandt D, K Müller, M Stech, KW Hilu, W Frey, JP Frahm, T Borsch 2004 Molecular evolution of the chloroplast $t r n L-F$ region in land plants. Monogr Syst Bot Mo Bot Gard 98:13-37.

Raubeson LA, RK Jansen 1992 Plastid DNA evidence on the ancient evolutionary split in vascular land plants. Science 255:1697-1699.

Renner SS 1998 Phylogenetic affinities of Monimiaceae based on cpDNA gene and spacer sequences. Perspect Plant Ecol Evol Syst 1: 61-77.

1999 Circumscription and phylogeny of the Laurales: evidence from molecular and morphological data. Am J Bot 86: 1301-1315.

Rodríguez F, JF Oliver, A Marín, JR Medina 1990 The general stochastic model of nucleotide substitution. J Theor Biol 142: 485-501.

Rohwer JG 2000 Toward a phylogenetic classification of the Lauraceae: evidence from matK sequences. Syst Bot 25:60-71.

Rokas A, BL Williams, N King, SB Carroll 2003 Genome-scale approaches to resolving incongruence in molecular phylogenies. Nature 425:798-804.

Ronse De Craene LP, DE Soltis, PS Soltis 2003 Evolution of floral structures in basal angiosperms. Int J Plant Sci 164(suppl): S329-S363.

Savolainen V, MW Chase, SB Hoot, CM Morton, DE Soltis, C Bayer, MF Fay, AY de Bruijn, S Sullivan, Y-L Qiu 2000 Phylogenetics of flowering plants based upon a combined analysis of plastid atpB and $r b c L$ gene sequences. Syst Biol 49:306-362.

Simmons MP, KM Pickett, M Miya 2004 How meaningful are Bayesian support values? Mol Biol Evol 21:188-199.

Soltis DE, VA Albert, V Savolainen, K Hilu, Y-L Qiu, MW Chase, JS Farris, JD Palmer, PS Soltis 2004 Angiosperm relationships, genome-scale data, and "ending incongruence": a cautionary tale in phylogenetics. Trends Plant Sci 9:477-483.

Soltis DE, AE Senters, MJ Zanis, S Kim, JD Thompson, PS Soltis, LP Ronse De Craene, PK Endress, JS Farris 2003 Gunnerales are sister to other core tricolpates: implications for the evolution of pentamery. Am J Bot 90:461-470.

Soltis DE, PS Soltis 2004 Amborella not a "basal angiosperm"? not so fast. Am J Bot 91:997-1001.

Soltis DE, PS Soltis, MW Chase, ME Mort, DC Albach, M Zanis, V Savolainen, et al 2000 Angiosperm phylogeny inferred from $18 \mathrm{~S}$ rDNA, $r b c L$ and $a t p B$ sequences. Bot J Linn Soc 133: $381-461$.

Soltis DE, PS Soltis, ME Mort, MW Chase, V Savolainen, SB Hoot, CM Morton 1998 Inferring complex phylogenies using parsimony: 
an empirical approach using three large DNA data sets for angiosperms. Syst Biol 47:32-42.

Soltis DE, PS Soltis, DL Nickrent, LA Johnson, WJ Hahn, SB Hoot, JA Sweere, et al 1997 Angiosperm phylogeny inferred from 18S ribosomal DNA sequences. Ann Mo Bot Gard 84:1-49.

Soltis PS, DE Soltis 1998 Molecular evolution of $18 \mathrm{~S}$ rDNA in angiosperms: implications for character weighting in phylogenetic analysis. Pages 188-210 in DE Soltis, PS Soltis, JJ Doyle, eds. Molecular systematics of plants. II. DNA sequencing. Kluwer, Boston.

Soltis PS, DE Soltis, MW Chase 1999a Angiosperm phylogeny inferred from multiple genes as a tool for comparative biology. Nature 402:402-404.

Soltis PS, DE Soltis, PG Wolf, DL Nickrent, S Chaw, RL Chapman $1999 b$ The phylogeny of land plants inferred from $18 \mathrm{~S}$ rDNA sequences: pushing the limits of rDNA signal? Mol Biol Evol 16:1774-1784.

Steel MA, PJ Lockhart, D Penny 1993 Confidence in evolutionary trees from biological sequence data. Nature 364:440-442.

Stefanovic S, DW Rice, JD Palmer 2004 Long branch attraction, taxon sampling, and the earliest angiosperms: Amborella or monocots? BMC Evol Biol 4, art. no. 35.

Steinhauser S, S Beckert, I Capesius, O Malek, V Knoop 1999 Plant mitochondrial RNA editing. J Mol Evol 48:303-312.

Suzuki Y, GV Glazko, M Nei 2002 Overcredibility of molecular phylogenies obtained by Bayesian phylogenetics. Proc Natl Acad Sci USA 99:16138-16143.

Swofford DL 1998 PAUP*4.0b10: phylogenetic analysis using parsimony. Sinauer, Sunderland, MA.

Tanaka N, H Setoguchi, J Murata 1997 Phylogeny of the family Hydrocharitaceae inferred from $r b c L$ and $m a t K$ gene sequence data. J Plant Res 110:329-337.
Taylor DW, LJ Hickey 1992 Phylogenetic evidence for the herbaceous origin of angiosperms. Plant Syst Evol 180:137-156.

Thien LB, H Azuma, S Kawano 2000 New perspectives on the pollination biology of basal angiosperms. Int J Plant Sci 161(suppl): S225-S235.

Thompson JD, TJ Gibson, F Plewniak, F Jeanmougin, DG Higgins 1997 The Clustal X windows interface: flexible strategies for multiple sequence alignment aided by quality analysis tools. Nucleic Acids Res 24:4876-4882.

Thorne RF 1992 An updated phylogenetic classification of the flowering plants. Aliso 13:365-389.

Ueda K, A Nakano, R Rodriguez, C Ramirez, H Nishida 1997 Molecular phylogeny of the Gomortegaceae, a Chilean endemic monotypic and endangered family. Not Biol 5:124.

Walker JW, JA Doyle 1975 The bases of angiosperm phylogeny: palynology. Ann Mo Bot Gard 62:664-723.

Wang X-Q, Y-Q Shu 2000 Plastid matK gene phylogeny of Taxaceae and Cephalotaxaceae, with additional reference to the systematic position of Nageia. Acta Phytotaxon Sin 38:201-210.

Wang X-Q, DC Tank, T Sang 2000 Phylogeny and divergence times in Pinaceae: evidence from three genomes. Mol Biol Evol 17: 773-781.

Williams JH, WE Friedman 2002 Identification of diploid endosperm in an early angiosperm lineage. Nature 415:522-526.

Zanis MJ, DE Soltis, PS Soltis, S Mathews, MJ Donoghue 2002 The root of the angiosperms revisited. Proc Natl Acad Sci USA 99: 6848-6853.

Zanis MJ, PS Soltis, Y-L Qiu, EA Zimmer, DE Soltis 2003 Phylogenetic analyses and perianth evolution in basal angiosperms. Ann Mo Bot Gard 90:129-150.

Zwickl DJ, DM Hillis 2002 Increased taxon sampling greatly reduces phylogenetic error. Syst Biol 51:588-598. 